\title{
VesselMap: A Web Interface to Explore Multivariate Vascular Data
}

\begin{abstract}
Hemodynamics plays a key role in the pathological evolution of vascular diseases such as vascular stenosis, plaques and aneurysms. Augmented visualization of relevant hemodynamic and geometric data accelerates engineering designs of diagnostic and therapeutic methods for those vascular diseases. However, four dimensional (4D) hemodynamic data are intrinsically complex. Consequently, exploration of this information has not been streamlined, despite its importance. In this paper, we propose a web interface to explore multivariate hemodynamic data, thereby facilitating the visualization of spatial and temporal relationships among those hemodynamic parameters of interest. The main thrust of this web system is the proposed VesselMap methodology, a 2D representation of vessel structures that provides an overview of all query results without any visual occlusions. Furthermore, in this framework, quantile-quantile plot was also used to compare spatial variations in parameter distributions, thereby greatly aiding in extracting local characteristics of hemodynamic parameters.

Using "patient-specific" computational fluid dynamics (CFD) simulations, realistic flow velocity fields were obtained. Based on those CFD-simulated velocity fields, we performed an empirical evaluation to confirm the usefulness of our approach for biomedical engineering applications. We concluded that the proposed VesselMap methodology allowed users to rapidly interact with hemodynamic data of interest, enhancing efficacy of information retrieval.
\end{abstract}

\section{Introduction}

2 Visualization of medical data has already been used extenз sively to show anatomical structures topologically using photo4 realistic surfaces or volume rendering. Moving forward, a more 5 integrated visualization approach is needed in order to quanti6 tatively connect processes of a disease with abnormalities at 7 various spatial and temporal levels in biomedical research. In8 tegral to this approach is an efficient way to retrieve quanti9 tative information from complex medical data. In this work, 10 visualization of vessel data in and around cerebral aneurysms 11 was used to demonstrate a novel web-based visualization ap12 proach of this kind. Vascular data in this paper are referring to 13 complex three-dimensional geometric data, hemodynamic data 14 (e.g., time-resolved 3D blood velocity) and other parameters 15 derived from hemodynamics.

${ }_{16}$ We envision that visual exploration of hemodynamic pa17 rameters and their spatial distributions facilitates engineering 18 design of therapeutic methods toward treating cerebral aneurysms.

19 Benefits of having such a web-based visualization tool are twofold

${ }_{20}$ First, the proposed tool provides a flexible but systematic way

${ }_{21}$ for biomedical engineers to explore flow data with their own

22 criteria. For instance, some vascular stents were designed to 23 open up arteries (i.e., structural integrity of vessel) while other 24 stents were designed to divert blood flow. Second, interdisci25 plinary studies of this kind often involve researchers located at ${ }_{26}$ different geographical locations. A web-based tool will enable 27 collaborative reviewing of experimental or computer simulation 28 results, reducing burdens on engineers for conducting a large29 scale study. Furthermore, we intend to provide an unobscured 30 viewing of $3 \mathrm{D}$ and $4 \mathrm{D}$ vessel data which may enhance the col${ }_{31}$ laborative reviewing process over the Internet. It is recognized 32 that labeling a $3 \mathrm{D}$ volume in a consistent way and communi- з3 cating it with peers over the Internet could be a difficult task, 34 because the relative positions of vessel branches may vary due 35 to the change of viewpoints. Now there are a large amount ${ }_{36}$ of time-resolved flow data spatially registered with the vessel 37 anatomy. Web-based collaborations involving in quantitative ${ }_{38}$ flow analysis without the right tools would be much more diffi39 cult.

Toward this end, we propose VesselMap, a novel web-based ${ }_{41}$ solution to assist biomedical experts in reviewing vessel data 42 and analyze the relationships among different properties of blood ${ }_{43}$ flow. The VesselMap is centered on a scheme that enables 2D 44 illustrative visualization of parameters for true 3D or 4D data. ${ }_{45}$ Using this scheme we first flatten the 3D vessel structure and ${ }_{46}$ its corresponding parameters onto a $2 \mathrm{D}$ plane. Then, all sub${ }_{47}$ sequent interactions can be operated in $2 \mathrm{D}$. This provides an ${ }_{48}$ easier way to specify and label regions of interest and elimi49 nates potential (angle-dependent) viewing occlusions. It also 50 serves as a clearer and paper-friendly overview, since rotation 1 and zooming are not available on a printed report. Furthermore, 52 a segmentation scheme is used to divide data into sub-groups ${ }_{53}$ for any parameters of interest, in order to provide comparative 54 investigations of flow characteristics. Data from sub-groups 55 generated from the aforementioned segmentation scheme can 56 be effectively displayed on the same 2D illustration, and their 57 inter-group differences are evaluated and displayed in a differ58 ence matrix. It is worth noting that an inherited benefit of the 59 proposed visual exploration method stems from the availability 60 of statistical information, such as histograms of different prop61 erties. Users can query flow characteristics within the entire 62 volume or a region of interest by simply interacting with his63 tograms. The proposed system is flexible and can be adapted 64 for different applications. Particularly, in this paper, some ben- 
65 efits of the aforementioned visualization strategy are going to be 66 demonstrated through visualization of particle transport in and 67 around cerebral aneurysms. Clinical applications include de68 liveries of chemical agents to either dissolve clots in the blood 69 stream [1], or occlude vascular aneurysms [2,3] and stop hem70 orrhage from ruptured aneurysms or arteries [4]. Flow visual71 ization is also important for designing flow-diverting stents to 72 occlude cerebral aneurysms or bleeding sites. Finally, the web73 based environment places a minimum amount of effort to setup 74 and requires only the displayed information to be transferred.

\section{2. Related Work}

76 Computed tomography angiography (CTA) is commonly 77 visualized with maximum intensity projection (MIP) and direct 78 volume rendering (DVR) techniques. To enhance the percep79 tion of vessel structures, researchers often approximate the ves80 sel surface using model-based or model-free surface rendering 81 approaches. The model-based surface rendering approach uti82 lizes the information of centerline and radius, and approximates 83 the vessel surface using models, such as truncated cones [5], B84 splines [6], subdivision surfaces [7], or convolution surfaces 85 [8]. The model-free surface rendering approach extracts the iso86 surface using algorithms such as marching cubes [9] based on 87 a given threshold. Instead of approximating the vessel surface, 88 Lathen et al. [10] proposed spatially varying transfer functions. 89 This approach locally shifts the transfer function to enhance the 90 perception of low intensity structures. Mistelbauer et al. [11] 91 used halo rendering to enhance the lumen of a vessel structure. ${ }_{92}$ Schumann et al. [12] used the multi-level partition of unity im${ }_{93}$ plicits (MPUI) approach to reconstruct the surfaces.

$94 \quad$ Unlike approaches based on MIP or DVR, other approaches 95 flatten the vessel structures and map the corresponding informa${ }_{96}$ tion to $2 \mathrm{D}$ images. One of the commonly used approaches in 97 this category is curved planar reformation (CPR). Kanitsar et 98 al. [13] introduced CPR as a curved cutting through the data 99 set along the centerline of a single vessel. Then, they extended 100 the CPR approach to multi-path CPR (mpCPR) that supports 101 multiple vessel branches and spiral CPR that flattens the vessel 102 along a spiral to show its interior [13]. Kretschmer et al. [14] 103 extended the mpCPR approach and used a bilateral filtering to 104 remove undesired depth discontinuities. Mistelbauer et al. [15] 105 proposed an approach based on CPR that aggregates the infor106 mation around the centerline along circular rays. Borkin et al. 107 [16] introduced a 2D vessel visualization method that uses a 108 tree diagram to represent the structure of a coronary artery tree. 109 Each branch is straightened and displayed as a tape with vary110 ing widths, which represents the diameter of the vessel. Zhu 111 et al. [17] presented a work that produces a flattened visual112 ization of vessel branches. Two algorithms were proposed in ${ }_{113}$ this work. The first one is a conformal mapping algorithm by 114 minimizing two Dirichlet functionals, and the second one ad115 justs the conformal mapping to produce a flattened representa116 tion that preserves areas. Marino and Kaufman [18] flattened 117 treelike structures based on their extracted skeletons. A ra118 dial planar embedding was adopted to layout the skeleton on a 119 plane. Neugebauer et al. [19] designed a multi-perspective 2D
120 projection map. Combined with a standard 3D visualization, a 121 correlation tool was provided to make correspondence between 122 the 2D map and 3D model. Won et al. [20] presented an un${ }_{123}$ cluttered single-image visualization of vascular structures The 124 centerlines were first extracted, so that the vascular structures 125 could be represented by multiple tubes. Each tube was then 126 laid out on 2D by minimizing a score function to avoid occlu127 sion. Instead of flattening the vessel structures, Neugebauer et 128 al. [21] selected appropriate viewpoints to reveal the anatomic 129 characteristics of cerebral aneurysms.

130 Other than scalar volumes, vascular data sets often come 131 with a simulated blood flow field as well. Recently, different 132 flow visualization techniques have been developed specifically ${ }_{133}$ for these data sets. van Pelt et al. [22] used various techniques 134 to depict the blood flow and associated characteristics in differ135 ent styles, together with an evaluation to measure the value of 136 those visualization styles. Köhler et al. [23] extracted vortices 137 in blood flow data sets using line predicates and highlighted the 138 corresponding regions. van Pelt et al. [24] proposed to semi139 automatically place and align a probe in the blood flow field, 140 which serves as a seeding basis. Then, particles, integral lines 141 and integral surfaces are used to convey distinct characteris142 tics of the flow field. Born et al. [25] found the representa143 tives of a line bundle, and used streamtapes with arrow heads 144 to visualize the line bundles. The tape-like structure provides 145 a clear picture of how the representatives diverge and merge. ${ }_{146}$ Oeltze et al. [26] proposed to cluster the streamlines and use 147 the cluster representatives for a clear view. They conducted a 148 qualitative study on using different similarity measures, includ149 ing geometry-based similarities and attribute-based similarities. 150 Angelelli and Hauser [27] presented a solution to straightening 151 tubular flow for side by side visualization, which facilitated the 152 comparison of different visualizations or time steps of the same 153 flow.

Due to the presence of multiple fields, some vessel visual155 ization approaches also provide contextual information. Straka 156 et al. [28] proposed VesselGlyph which combines both DVR 157 and CPR. It depicts the vessels using CPR which is naturally 158 placed in a DVR anatomic context. Mistelbauer et al. [11, 15] 159 provided optional context rendering that displays the volume 160 outside the lumen of vessel as well. Gasteiger et al. [29] pre161 sented a focus+context approach called FlowLens that uses some 162 predefined lens templates to combine visualization results of 163 different properties. The property of focus and property in con164 text are both selected by the users. Lawonn et al. [30] intro165 duced an adaptive surface visualization of vessels to highlight 166 the animated pathlines and emphasize nearby surface regions. ${ }_{167}$ Lawonn et al. [31] combined the blood flow animation with 168 wall thickness visualization. The wall thickness was mapped 169 onto the vessel surface, which could be cut away dynamically 170 to display a glyph-based blood flow animation.

Among the above approaches, those that flatten the vessel 172 and produce 2D images are closest to our approach. However, 173 unlike those approaches, our 2D visualization serves not only 174 as an overview but also as an interface for convenient interac175 tion. We provide very concise information on the 2D visual176 ization as a guideline to explore the data. Our approach does 
177 not require the centerline and radius information as CPR-based 178 techniques $[32,13,15]$, the triangulated vessel surface as the 179 conformal mapping technique [17], or others do [20, 18]. In 180 addition, these approaches only visualize scalar information of 181 the vessel, without providing the ability to trace particles in the 182 vessels, which is important for discover hemodynamic char183 acteristics, such as particle transport information. In terms of ${ }_{184}$ exploring multiple fields, our approach also differs from those 185 focus+context techniques [28, 29]. Instead of visualizing mul186 tiple fields, we provide an interface guided by histograms of dif187 ferent properties to enable users to discover the dynamic statis188 tical information for regions of interest during particle tracing. 189 It also facilitates the process of finding a region with a certain 190 feature, or exploring relationships among different regions.

\section{${ }_{191}$ 3. Algorithm}

192 Our exploration is guided by statistical information, mostly 193 histograms of particles with different properties. The property 194 values of particles are either interpolated in scalar volumes, e.g., 195 pressure and wall shear stress (WSS), or derived from particle 196 tracing, e.g., age. Users can freely interact with the histograms 197 to select a combination of bins in one or more histograms to 198 filter particles and highlight the corresponding regions. In ad199 dition, we propose an approach to map the 3D vessels to a $2 \mathrm{D}$ 200 VesselMap, while preserving its perceptual structure. Users can 201 brush the 2D VesselMap or select a group from our segmen202 tation to specify a region of interest, and the histograms com203 putation will be constrained to the user-specified region. The 204 viewpoint of the 3D particle rendering can be automatically ad205 justed to focus on that region and reveal more details in the 206 original 3D space. Users can further interact with histograms 207 or select regions to gradually refine their query. They can also 208 brush the 2D VesselMap to place a seeding plane from which 209 the particles will first be released and then be traced.

210 We provide a segmentation of the vessel structure to com211 pare the histograms in different regions, so that local behavior 212 can be observed. The segmentation can be guided by differ213 ent mapping functions to provide a variety of results. The dif214 ferences among regions are derived from their histograms. In 215 addition to the traditional measures which usually compute a ${ }_{216}$ single value to represent the difference, we visualize the differ217 ences using quantile-quantile (Q-Q) plots. For global investiga218 tion, we develop an interface that shows all differences between 219 every pair of groups.

${ }_{220}$ Our system consists of a web front-end client for visual221 ization and user interface, and a back-end server for compu222 tation. The server is responsible for histogram computation, 223 VesselMap generation, and flow visualization. While the web 224 front-end displays visualization results, it also interacts with 225 users and requests the server to update visualization results us226 ing messages.

\section{3.1. VesselMap: a $2 D$ Representation}

228 The entire volume is evenly partitioned into small blocks 229 (e.g., $3 \times 3 \times 3$ in this paper), and only blocks containing the 230 vessel structure are loaded. These $3 \mathrm{D}$ blocks approximate the (a)

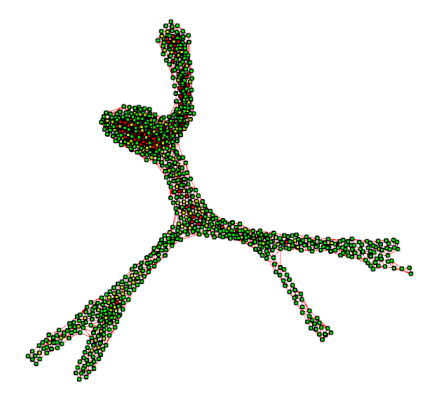

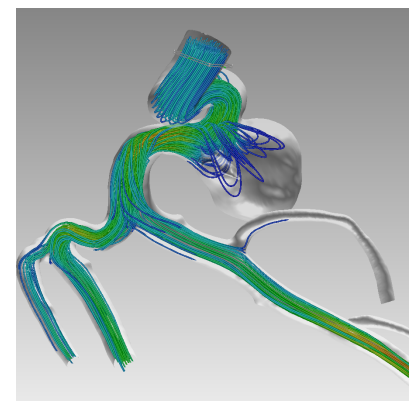

(b)
Figure 1: Mapping from the 3D volume space to a 2D VesselMap representation using the VDS1 data set. (a) shows the mesh structure of the VesselMap. (b) shows streamline visualization with a semi-transparent vessel structure.

231 vessel structure. We map the blocks to points in $2 \mathrm{D}$, so that they 232 can be displayed on a webpage more desirably. The mapped 233 points are then triangulated to form a mesh structure represent234 ing the vessel structure. To build the connection between the 235 original $3 \mathrm{D}$ volume and the $2 \mathrm{D}$ image, the mapping should pre236 serve the local shape of vessels. We formulate this as a $2 \mathrm{D}$ 237 graph layout problem, and achieve the desired layout using a 238 minimization approach. Assuming the blocks that contain some 239 of the vessel structure are $B=\left\{b_{1}, b_{2}, \cdots, b_{n}\right\}$, the neighboring 240 blocks of $b_{i}$ are those whose distances to $b_{i}$ are smaller than or 241 equal to a given threshold $\delta$, and the non-neighboring blocks 242 are those whose distances to $b_{i}$ are larger than $\delta$. The energy of 243 the mapping is defined as follows:

$$
\sum_{b_{i} \in B} \sum_{b_{j} \in B} c_{i j}, \text { where } \quad c_{i j}=\left\{\begin{array}{l}
w_{n}\left|d_{i j}-e_{i j}\right|, e_{i j} \leq \delta \\
w_{n n}\left|d_{i j}-g_{i j}\right|, e_{i j}>\delta .
\end{array}\right.
$$

${ }_{245}^{244}$ where $c_{i j}$ is the cost between $b_{i}$ and $b_{j}, w_{n}$ and $w_{n n}$ are weights 246 for neighboring and non-neighboring blocks, respectively, $d_{i j}$ 247 is the distance between $b_{i}$ and $b_{j}$ in the 2D image, $e_{i j}$ is their ${ }_{248}$ Euclidean distance in the original 3D volume and $g_{i j}$ is their 249 geodesic distance in the 3D vessel structure. Intuitively, for 250 neighboring blocks, we preserve their original distances to main251 tain the local shape of vessels. For non-neighboring ones, using 252 the geodesic distance will separate two blocks in different ves253 sel branches to avoid occlusion. Figure 1 (a) shows the mapped 254 points with triangulation, we observe that the shape is still sim255 ilar to that in $3 \mathrm{D}$, but occlusion is removed. Each point is dis256 played as a node whose color represents the distance from the 257 corresponding data block to the vessel wall. A block that is 258 in proximity of the vessel is colored in green. The color tran259 sits from green to red for blocks further away from the vessel 260 wall. Since the widths for most vessel branches are small when 261 measured in number of blocks, we can only observe a few dif262 ferent colors. Visually, we find that red nodes are likely to stay 263 around the centerline of the flattened vessel, consistent with our 264 intuition. This indicates visualization through the flattened Ves265 selMap can still be used to infer spatial characteristics, although 266 spatial continuities among nodes is no longer valid.

267 Considering block centers as point clouds, we approximate 268 the geodesic distance using the shortest path between two points. ${ }_{269}$ Given a threshold $\varepsilon$, we initialize the geodesic distance matrix 270 as follows: 


$$
g_{i j}=\left\{\begin{array}{l}
e_{i j}, e_{i j} \leq \varepsilon \\
\infty, \text { otherwise. }
\end{array}\right.
$$

${ }_{272}^{271}$ where $e_{i j}$ is the Euclidean distance between the centers of blocks ${ }_{273} b_{i}$ and $b_{j}$. Running an all-pair shortest distance algorithm, e.g., 274 the Floyd-Warshall algorithm, on this distance matrix, we can 275 obtain the approximated geodesic distance in $O\left(n^{3}\right)$ time, where $276 n$ is the number of blocks. In our implementation, we use ${ }_{277} \varepsilon=1.5$, meaning that at the initial stage, only the distances 278 between neighbors are known, and the distances between non279 neighboring blocks are then approximated by the shortest paths.

To obtain the positions of vertices $V=\left\{v_{1}, v_{2}, \cdots, v_{n}\right\}$, where $v_{i}$ is a $2 \mathrm{D}$ vertex that represents block $b_{i}$, the equations are represented as a linear system, so that the minimization is performed by solving this linear system in a least-squares sense. Since in these equations $d_{i j}=\sqrt{\left(v_{i}-v_{j}\right)^{2}}$ is not linear, we propose a scheme to solve them in multiple iterations. Let $V=$ $\left\{v_{1}, v_{2}, \cdots, v_{n}\right\}$ be the desired positions and $V^{\prime}=\left\{v_{1}^{\prime}, v_{2}^{\prime}, \cdots, v_{n}^{\prime}\right\}$ be the current positions. Then a target distance $t_{i j}$ is achieved by moving the two vertices along the line segment connecting them. Note that the target distance $t_{i j}$ is $e_{i j}$ for neighboring blocks and $g_{i j}$ for non-neighboring blocks. In this way, the equation $\left|d_{i j}-t_{i j}\right|$ is rewritten in the form of

$$
\left|\left(v_{i}-v_{j}\right)-t_{i j}\left(v_{i}^{\prime}-v_{j}^{\prime}\right) /\left(\left|v_{i}^{\prime}-v_{j}^{\prime}\right|\right)\right|
$$

${ }_{280}$ Note that $v_{i}$ and $v_{j}$ are variables to solve, and $t_{i j}\left(v_{i}^{\prime}-v_{j}^{\prime}\right) /\left(\mid v_{i}^{\prime}-\right.$ $\left.281 v_{j}^{\prime} \mid\right)$ is a constant. Therefore, the equation is a linear combina282 tion of the variables. All equations can then be formulated in a 283 linear system $A V=C$, where $A$ is an adjacency matrix, $V$ is a 284 vector of all vertices, and $C$ is a vector of all constants. Each 285 equation is represented by one row in the linear equation. For 286 example, assume that $\left(v_{i}-v_{j}-c\right)^{2}$ is the $k$-th equation. Then 287 the $k$-th row in matrix $A$ is constructed as the following: its $i$-th 288 element is set to 1 , its $j$-th element is set to -1 , and all the other 289 elements are set to 0 . And the $k$-th element in the vector $C$ is set 290 to $c$. Applying the least square method will provide us a new 291 set of positions based on the current positions. We will repeat 292 this procedure until the positions do not change or some prede293 fined number of iterations is achieved. In our implementation, 294 if the change of energy is smaller than $1 \times 10^{-6}$, the positions 295 are considered to be stable, with the maximum number of iter296 ations being 1500 .

Viewpoint selection. When users brush a group of blocks to query, the viewpoint of the $3 \mathrm{D}$ rendering can be changed accordingly to focus on that region. We adopt a heuristic scheme to automatically select the viewpoint. Each block is considered as a data point located at the center of that block. We compute three principal components of the data points, and the variations along all principal component directions. The viewing direction is given by

$$
\operatorname{var}_{3} \cdot p_{1}+\operatorname{var}_{2} \cdot p_{2}+\operatorname{var}_{1} \cdot p_{3},
$$

297 where $p_{1}, p_{2}$ and $p_{3}$ are the first, second and third principal 298 component directions, and $v a r_{1}, v_{2} r_{2}$ and $v a r_{3}$ are the variations 299 along the three directions, respectively. Intuitively, if the vari300 ation of point positions along one direction is large, the view$301 \mathrm{ing}$ direction is desired to be perpendicular to that direction in

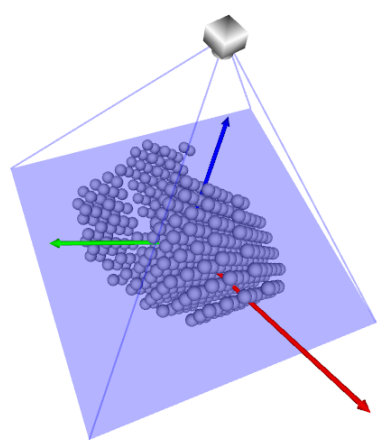

(a)

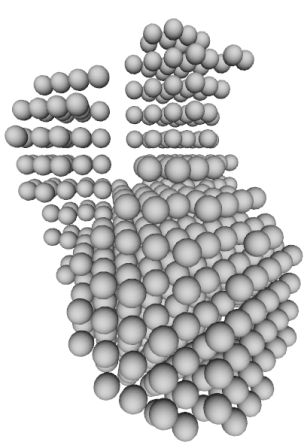

(b)
Figure 2: The automatic viewpoint selection when users brush the $2 \mathrm{D}$ VesselMap. (a) shows the selected blocks and their principal components together with the camera. (b) shows the rendered result under the automatically selected viewpoint.

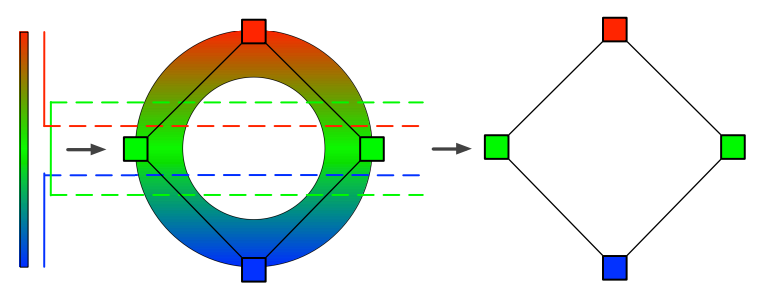

Figure 3: The nerve of a covering using a torus as an example. The torus is colored by a height function. This set of images demonstrates how a covering of the height function becomes a covering of the torus, whose nerve captures the topology of the torus with one connected component and a hole.

302 order to preserve the variation. Therefore, the three principal 303 component directions are weighted by their variations in the re304 versed order, so that the most significant component gets the 305 smallest weight to increase its angle difference from the view306 ing direction. In Figure 2 (a), each block is represented by a 307 sphere located at its center. The first, second and third princi308 pal components are indicated by the red, green and blue arrows, 309 whose lengths are proportional to the variations along those di310 rections. Since the variations along all directions are similar 311 to each other, the camera tends to look at them in a balanced 312 way. The projected plane is shown by the blue rectangle, whose 313 center is determined by averaging the center positions of all se314 lected blocks. The rendering result under the automatically se315 lected viewpoint is shown in Figure 2 (b).

316 Seeding plane placement. In this application, all parti317 cles are released from an inlet plane specified by a user-defined 318 plane in a vessel branch. Our 2D VesselMap can be used to 319 place the seeding plane conveniently. Users can simply brush 320 the VesselMap to select a group of blocks, and the seeding plane 321 will be placed at the center of those blocks. While the size of 322 the seeding plane is specified by the users, the normal direction 323 is given by the flow direction in order to maximize the effective 324 area for particle releasing.

\section{3.2. VesselMap Segmentation}

In the next stage, the VesselMap is segmented into regions. 327 Inspired by Singh et al. [33], we develop this segmentation 328 scheme to divide the vessel structure at the block level based 329 on a mapping function that assigns each block a scalar value. 


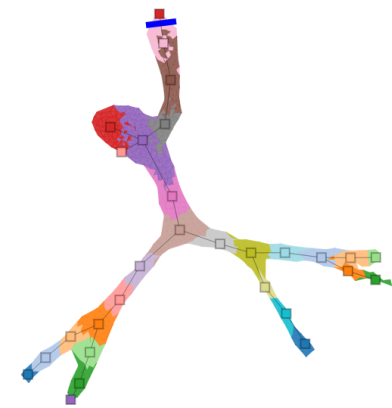

(a)

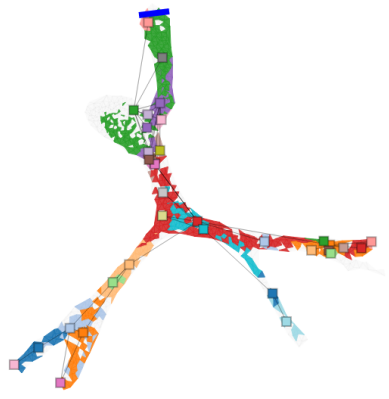

(b)
Figure 4: The segmentation and the nerve of the VesselMap using the geodesic distance to the inlet as the mapping function. The result is colored by groups of blocks. The rectangle nodes and the edges connecting them show the nerve of the VesselMap. (a) Blocks are grouped by their distances to the inlet. (b) Blocks are grouped by the vorticity values of the particles. In this case, the blocks that do not contain any particle are not grouped and are colored in white.

${ }_{330}$ The regions generated from the segmentation serve as a start331 ing point to investigate their local behaviors. The connection 332 among the segmented regions forms what is known as the nerve $3{ }_{33}$ of the VesselMap. The nerve of the VesselMap provides an ab334 stract overview of the vessel data. Depending on the selection 335 of the mapping function, the nerve of the VesselMap conveys $3 з 6$ different information. For example, if the geodesic distance 337 from a block to the inlet is used, it represents the shape of the 338 vessel structure; while if a scalar property in a block is used, $3 з$ the nerve of the VesselMap represents the topological structure 340 of that property.

341 Background. The construction described in this section is 342 motivated by the concept of the nerve of a covering in the field 343 of topology. Given a scalar field over the domain, this provides 344 an abstraction of how different value ranges of the scalar field 345 are related to each other. We refer readers to [34] for back346 ground information on topological space and simplicial com347 plex, and only provide an intuitive description here with an ex348 ample. In this example, we construct the nerve of a covering 349 that captures the topology of a torus. A finite covering is a col350 lection of subsets whose union is the entire space. In the left 351 part of Figure 3, an interval $I \in \mathbb{R}$ is colored from red to green 352 to blue, and three smaller intervals are indicated by three line 353 segments to its right: the red one at the top, the green one in 354 the middle, and the blue one at the bottom. The three small 355 intervals represented by these three lines form a covering of $I$, 356 since their union covers the entire interval of $I$. However, there 357 is no such covering immediately available for a torus. In this 358 example, we obtain the covering of the torus through the cov359 ering of an interval. Consider the height function of the torus ${ }_{360} f: T \rightarrow \mathbb{R}$, that maps each point of the torus $T$ to a scalar value 361 of height. We see that the covering of the range of this height 362 function naturally becomes a covering of the torus, with a blue 363 region at the bottom, two green regions in the middle, and one 364 red region on the top. The nerve of this covering is then gen365 erated by connecting the overlapping regions. The right part of ${ }_{366}$ Figure 3 shows that the nerve captures the topology of the torus.

Our construction. Similarly, we obtain a covering of the 368 blocks $B$ by considering a map $f: B \rightarrow \mathbb{R}$, which assigns each 369 block a real number. This map could be the distance to the

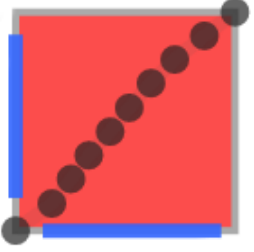

(a)

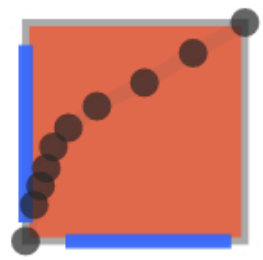

(b)

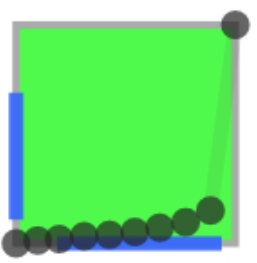

(c)
Figure 5: Q-Q plot examples. The correlation of quantiles is mapped onto the background color of the plot. The ranges of quantiles are mapped by two blue bars. The two distributions in (a) are very similar; those in (b) are less similar; and those in (c) are least similar.

370 inlet plane, or the average velocity magnitude in a block, etc. 371 Assuming the range of $f$ is $\left[r_{\min }, r_{\max }\right]$, the subsets $\left\{\left[r_{\min }, r_{1}\right]\right.$, $\left.372\left[r_{2}, r_{3}\right], \ldots,\left[r_{k}, r_{\max }\right]\right\}$ is a covering of $\left[r_{\min }, r_{\max }\right]$, and $\left[r_{i-1}, r_{i}\right]$ 373 and $\left[r_{i+1}, r_{i+2}\right]$ are two overlapping subsets. Considering the in374 terval $[0,10]$ as an example, $\{[0,4],[3,7],[6,10]\}$ is such a cov375 ering of $[0,10]$. In our implementation, we first determine the 376 minimum and maximum of the mapped values, i.e., $r_{\min }, r_{\max }$. 377 Then, we evenly divide the range into overlapping sub-ranges, 378 and each block corresponds to one or more sub-ranges accord379 ing to its mapped value. Finally, connecting the neighboring 380 blocks that correspond to the same sub-range forms a set of 381 connected components, which serve as the vertices in the nerve 382 of the VesselMap, denoted as $V=\left\{v_{1}, v_{1}, \ldots, v_{m}\right\}$, where $m$ is 383 the number of generated connected components. We name each 384 connected component a group of blocks. Note that the connec385 tion relationship is computed from 3D blocks. Therefore, the 386 topological structure will not be affected by the mapping from 387 BD to 2D.

Although the nerve of a covering may contain higher di389 mensional simplices, for the nerve of the VesselMap, we only 390 consider simplices up to dimension one, i.e., vertices and edges. 391 Therefore, only the connections between two vertices are taken 392 into consideration. Following the previous definition, an edge 393 (1-simplex) $e_{i j}=\left(v_{i}, v_{j}\right)$ is in the nerve of a covering, if and 394 only if $U_{i} \cap U_{j} \neq \emptyset$, where $U_{i}$ and $U_{j}$ are subsets in the covering 395 corresponding to $v_{i}$ and $v_{j}$, respectively. In our context, if two 396 groups of blocks share some blocks in common, an edge will 397 be added between them in the nerve of the VesselMap.

398 However, this procedure generates segmentation results with 399 overlapping. We further simplify the procedure and divide the 400 range of $f$ into non-overlapping sub-ranges, and therefore, the 401 groups are non-overlapping as well. In this case, an edge $e_{i j}=$ $402\left(v_{i}, v_{j}\right)$ is in the nerve of the VesselMap, if a group represented 403 by $v_{i}$ contains a block $b_{p}$ that is a neighbor of some block ${ }_{404} b_{q} \in v_{j}$. Figure 4 shows two examples of the segmentation and 405 the nerve of the VesselMap. In Figure 4 (a), the neighboring 406 blocks whose geodesic distances to the inlet fall into the same 407 range are grouped together. This segmentation produces similar 408 results as those segmentation approaches considering the shape 409 of the structure. In addition, the corresponding nerve demon410 strates the topological structure of the vessel.

\section{3.3. Comparing Regions and Properties}

412 One of the major goals of our application is to guide users ${ }_{413}$ to explore the relationships among properties. However, we 


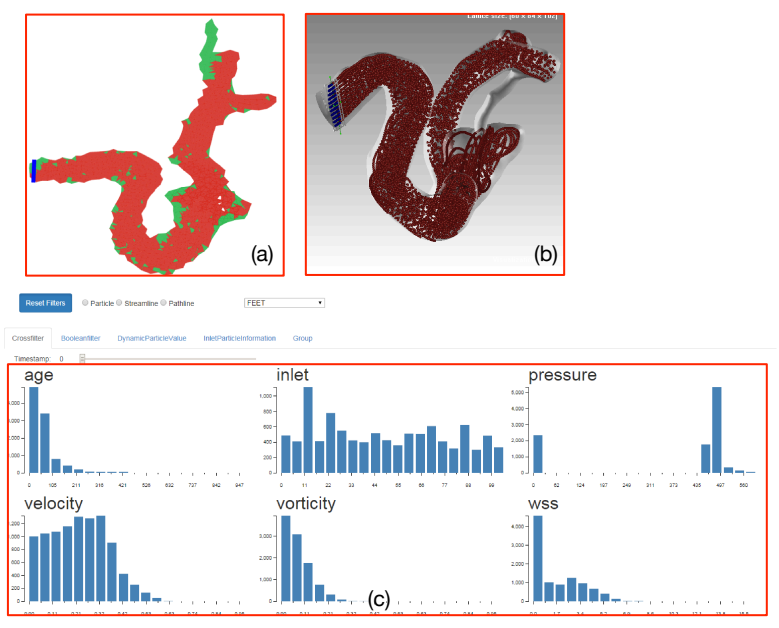

Figure 6: The VesselMap Interface. (a) shows the 2D VesselMap. (b) shows the $3 \mathrm{D}$ flow visualization. (c) shows the histogram visualization, which is a tab of the information interface.

${ }_{414}$ feel that the commonly used approaches, e.g., correlation co415 efficients, only provide one single value indicating the differ416 ence, which fails to answer how they differ from each other. ${ }_{417}$ In our approach, in addition to the traditional difference val418 ues, we use the Q-Q plot [35], to capture the details. Quantiles 419 are points taken at regular intervals from the cumulative density 420 function $(\mathrm{CDF})$ of a random variable. The concept of quantiles 421 differs from percentiles in the sense that quantiles are indexed 422 by sample fractions instead of sample percentages. Generally, ${ }_{423}$ the $p$-th quantile $Q(p)$ of a random variable $Z$ is the value $z$ 424 such that $P(Z \leq z)=p$. The Q-Q plot for two random vari425 ables $Z_{1}$ and $Z_{2}$ plots the quantile pairs $<Q_{Z_{1}}\left(p_{1}\right), Q_{Z_{2}}\left(p_{1}\right)>$ ${ }_{426},<Q_{Z_{1}}\left(p_{2}\right), Q_{Z_{2}}\left(p_{2}\right)>, \cdots,<Q_{Z_{1}}\left(p_{n}\right), Q_{Z_{2}}\left(p_{n}\right)>$ on a $2 \mathrm{D}$ plane 427 where $p_{1}<p_{2}<\cdots<p_{n}$ are equally spaced.

${ }_{428}$ Figure 5 shows three examples of the Q-Q plot. The back${ }_{429}$ ground color of a Q-Q plot represents the correlation of quan430 tiles for the two corresponding groups. The green, brown and ${ }_{431}$ red colors indicate small, medium and large correlations, re432 spectively. The color gradually transits in between. For a clearer 433 view, the plotted points always occupy the entire space, i.e., 434 they start from the bottom-left corner and end at the top-right 435 corner. In addition, a blue bar is used to indicate the local value 436 range in a group with respect to the global value range in the ${ }_{437}$ volume. In (a), the two distributions are almost identical and 438 the points are mostly aligned along the diagonal. In (b), the two ${ }_{439}$ distributions are less similar. The slope of the Q-Q plot is first 440 steep and then becomes flat. This indicates that the distribution 441 corresponding to the $x$-axis has slightly higher probabilities for 442 the smaller values while the distribution corresponding to the ${ }_{443} y$-axis has slightly higher probabilities for the larger values. In ${ }_{444}$ (c), the two distributions are least similar. The slope of the Q-Q 445 plot is almost flat for a large portion of quantiles. This indicates 446 that the distribution corresponding to the $y$-axis has very high 447 probabilities for the smallest values. Thus, a Q-Q plot provides 448 more information on how the two distributions differ from each 449 other.

\section{4. User Interface and Interaction}

451 Our interface is shown in Figure 6. It consists of three com452 ponents: the VesselMap in the top right region, the flow visu453 alization in the top left region, and the information interface at 454 the bottom. The VesselMap displays the 2D representation of 455 the vessel structure. A blue bar is shown to indicate the inlet 456 where particles are released, as shown in Figure 7 (b). If his457 togram, particle inlet information, or particle stay information 458 is displayed, the triangles are colored in red or green: red in459 dicates blocks in the triangle containing queried particles, and 460 green indicates blocks in the triangle containing no queried par461 ticles. When group comparison is displayed, the triangles are 462 colored according to the groups they belong to. Flow visualiza463 tion displays the vessel surface as a semi-transparent mesh and 464 the flow inside the vessel, as shown in Figure 7 (c). The flow 465 can be visualized as particles, streamlines or pathlines. Flow 466 visualization is computed by the server, and only the resulting 467 images are sent to the webpage. Users can select six orthogonal 468 directions, i.e., head, feet, anterior, posterior, left and right, to 469 view the data, or a predefined direction which is specified on 470 the server. The information interface contains four tabs: cross 471 filter, boolean filter, particle inlet visualization, and group com472 parison. Cross and boolean filters both contain histogram visu473 alizations, where users can interact with the histograms to filter 474 the particles and highlight regions in the 2D VesselMap. Parti475 cle inlet visualization shows the particle distribution over the in476 let plane that feeds the user-specified region. Note that a single 477 seeding plane is used as the inlet of particles. When referring to 478 a property of particles, we use the term "particle inlet", or sim479 ply "inlet", to indicate the position where a particle is released 480 in the seeding plane, instead of a specific inlet among multiple ${ }^{481}$ inlets. Group comparison demonstrates the differences among 482 groups of blocks generated from the segmentation.

\section{${ }_{483}$ 4.1. Histogram Visualization and Filtering}

484 Our method visualizes statistical information of different 485 properties, such as velocity, vorticity, WSS, particle inlet and 486 pressure, in real time. Users can specify a region of interest 487 where the histogram will be generated. If it is not specified, 488 the entire volume will be taken into consideration. As shown 489 in Figure 7 (a), the histograms are visualized as bar charts and 490 the selection of a certain range of property values to further re491 fine the histogram computation can be directly performed on 492 those bar charts. This will be very helpful for domain experts, 493 since finding the regions that meet certain criteria is a common 494 task, e.g., finding the region with high pressure and low WSS. 495 We provide two methods to specify the refining query criteria: 496 cross filter and boolean filter.

For cross filter, users can mouse over multiple bins of a his498 togram, so the computation of all other histograms will be re499 stricted to the particles falling into these selected bins. In other 500 words, when computing other histograms, only the particles in 501 these selected bins are counted. If multiple histograms are se502 lected, the computation of all non-selected histograms will be 503 restricted by these histograms; and any selected histogram will 504 be restricted by other selected ones but not by itself. The first 

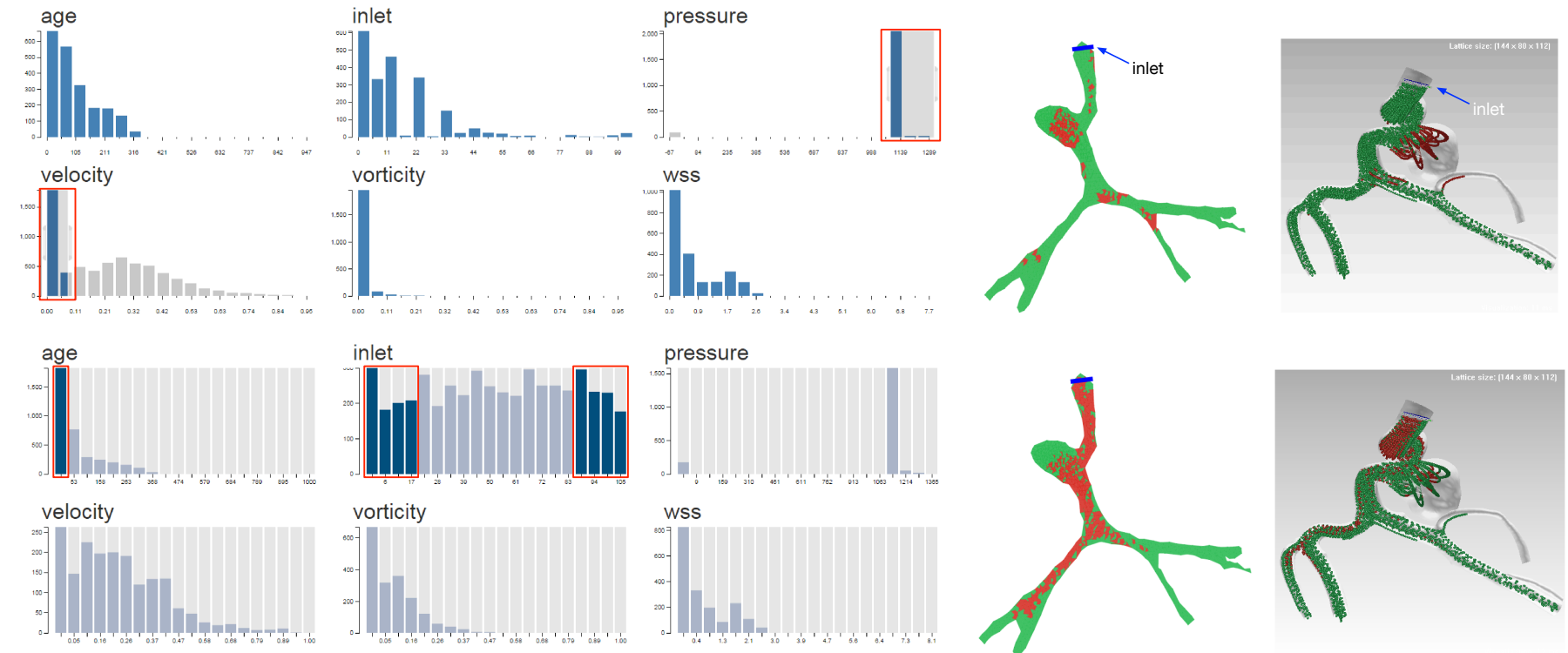

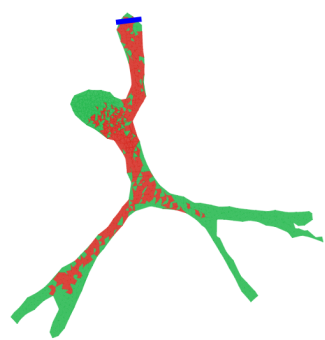

(b)

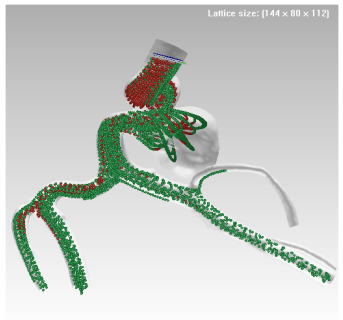

(c)

Figure 7: Cross filter and boolean filter for refining queries on histograms. The selected ranges in the histograms are highlighted in red rectangles. Column (a) shows the interface of visualizing histograms and selection of bins, where selection is performed by a cross filter in the first two rows and a boolean filter in the later two rows. Column (b) shows the highlighting results on the VesselMap, where red blocks contain particles fulfilling the selection criteria and the green ones do not. Column (c) shows the highlighting results of particles in the original 3D volume.

505 row of Figure 7 shows an example of a cross filter. In (a), the 537 map corresponds to a position on the inlet plane. This plane is 506 bins corresponding to lower velocity and higher pressure are se- 538 displayed as a blue bar in the VesselMap, as shown in Figure 507 lected using a cross filter. Histograms of other properties show 5397 (b). In flow visualization, the plane is shown as a transpar508 the distributions of particles with low velocity and high pres- 540 ent box with white boundaries, and the particles on the plane 509 sure values, the velocity histogram shows the distribution of 541 are colored in blue, as shown in Figure 7 (c) pointed by the la510 particles with high pressure values, and the pressure histogram 542 bel "inlet". The seeding plane is evenly partitioned into small 511 shows the distribution of particles with low velocity. The blocks 543 regions. Each small region corresponds to a color block on the 512 and particles are also highlighted according to the selected bins, 544 heat map. The color indicates the number of particles originated 513 as shown in (b) and (c), respectively. Those particles that be- 545 from the corresponding region which are currently in the region 514 long to the selected bins are highlighted in red, while other par- 546 of interest. Users can interact with the VesselMap to specify the 515 ticles are displayed in green.

516 The boolean filter behaves similarly. Nevertheless, instead 517 of brushing across the bins, users need to click on multiple bins 518 to specify the refining criteria. Considering the selection status 519 of each bin as a boolean, the selected bins in the same histogram 520 are connected by the or operator, while those in different his521 tograms are connected by the and operator. The constraints on 522 different histograms are the same as with the cross filter. The 523 second row of Figure 7 demonstrates an example of a boolean 524 filter. Eight bins of inlet are selected, which means only par525 ticles released from these eight positions will be counted. In 526 addition, a bin of age is selected to further constrain that only 527 the particles with small age are counted.

528 Note that using cross filter, users can select a portion of the 529 range of a bin, and the corresponding value will be interpolated. ${ }_{530}$ But they are not able to select two disjoint ranges for query. ${ }_{531}$ Using boolean filter, users can select disjoint ranges. But for ${ }_{532}$ each selected bin, the entire range of the bin is used.

\section{5зз 4.2. Particle Inlet Visualization}

534 Particle inlet visualization displays the number of contribut535 ing particles in a user-specified region for each inlet position us536 ing a heat map. In our case, each small color block on the heat
547 region of interest, as shown in Figure 8. Note that some blocks 548 in the selected region are still colored in green because these 549 blocks do not contain any particle. Figure 8 shows two heat 550 maps with respect to the aneurysm and a segment of a vessel 551 branch, respectively. This information might help biomedical 552 engineers identify the vessel branches that feed the aneurysm 553 and the locations to release drugs that will be delivered to the 554 desired location. For example, in the first row of Figure 8, we 555 find that most particles in the aneurysm region are released from 556 the upper right region of the seeding plane.

\section{4.3. Visualization of Particle Transportation Information}

558 Visualization of particle transportation is important for clin559 ical applications. More specifically, therapeutic outcomes could 560 be better predicted if we can reliably assess how those chemi$561 \mathrm{cal} / \mathrm{biological}$ agents interact with the blood stream, perhaps, 562 in a "patient-specific" manner. Using a cerebral aneurysm as 563 an example, users can specify a duration of time during which 564 chemical agents or particles are released at different locations, 565 as shown by the blue and orange line segments in Figure 9 (a). 566 Then, particle transportation will be assessed and recorded be567 tween two time-intervals from the first particle being released 568 to the last particle leaving the volume of interest. As shown in 

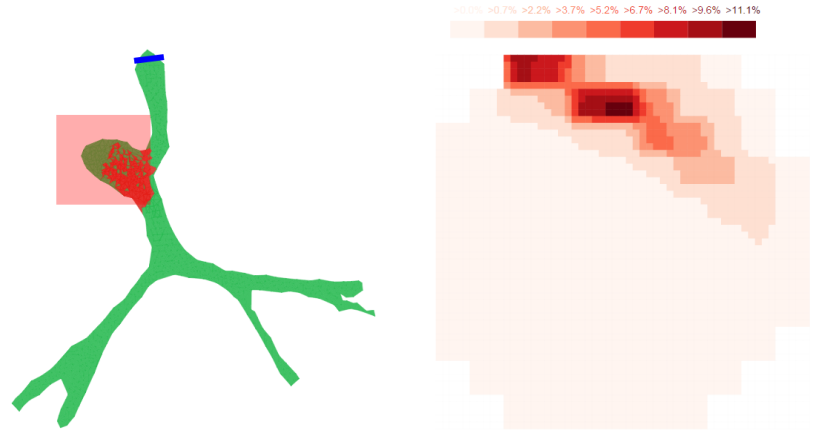

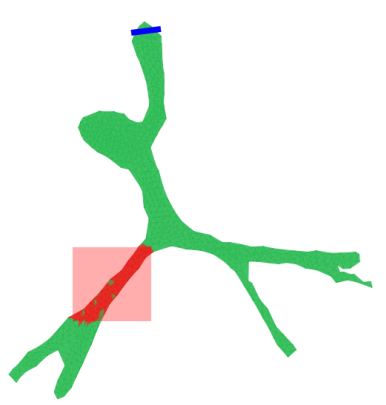

(a)

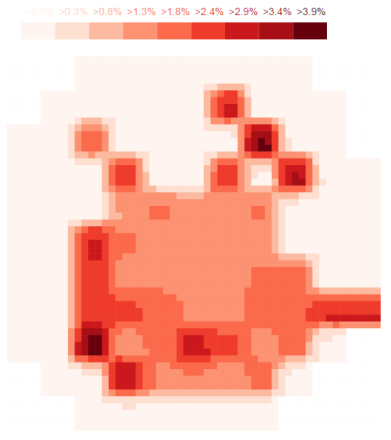

(b)
Figure 8: Contributing particle distribution at a flow inlet. (a) shows the userselected region on the VesselMap. (b) is the heat map that indicates the number of contributing particles released from each position on the seeding plane. In the first row, the region of interest is the aneurysm. In the second row, the region of interest is a segment of a vessel branch highlighted in red.

569 Figure 9 (b), time-intensity curves related to agent release can 570 be stored and analyzed. Therefore, clinically-relevant parame571 ters such as peak (particle) density, time to peak, and washout 572 time can be obtained. In addition, we display the histogram of 573 lengths of time that each particle stays in the selected region, as 574 shown in Figure 9 (c) and (d). Our system can store multiple 575 curves for comparison. In Figure 9 (b), a comparison between 576 two releasing locations is provided. It is easy to imagine that 577 those catheter-based therapies [36] would often become more 578 effective if those injected agents would arrive in a large quantity 579 and remain in the wounded site longer. By examining multiple 580 releasing locations like that, it would help clinicians optimize 581 the effects of agent delivery.

\section{4.4. Group Comparison}

583 In the segmentation, the vessel structure is divided into sev584 eral groups of blocks guided by some property. The group com585 parison interface is designed to investigate the differences of 586 histograms among these groups. Note that the property for his587 togram computation is specified by the users, and could be dif588 ferent from the one that guides the segmentation. In this way, 589 the local behaviors and relationship between properties can be 590 better studied. The differences are organized in an $n \times n$ matrix, 591 where each cell visualizes a difference value together with a $Q$ ${ }_{592} \mathrm{Q}$ plot indicating how the two distributions differ. All groups 593 are sorted by decreasing numbers of particles in them, and ar594 ranged left to right and top to bottom in the matrix. Figure 10 595 (a) shows an example of the difference matrix interface. Users 596 can use this interface to narrow down a region of interest. By

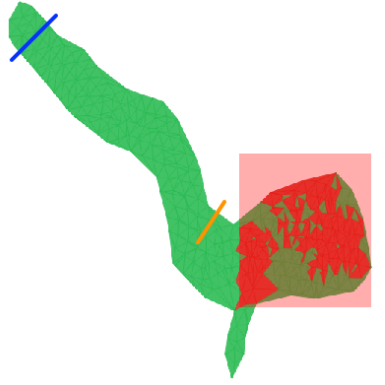

(a)

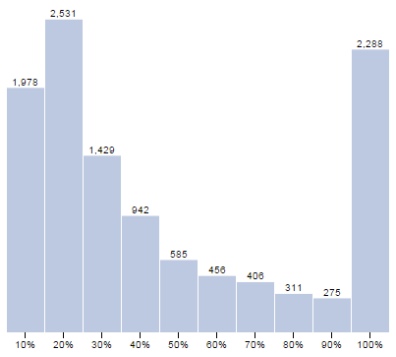

(c)

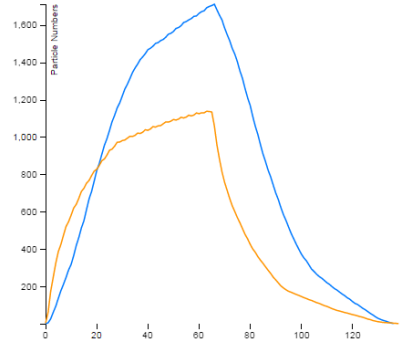

(b)

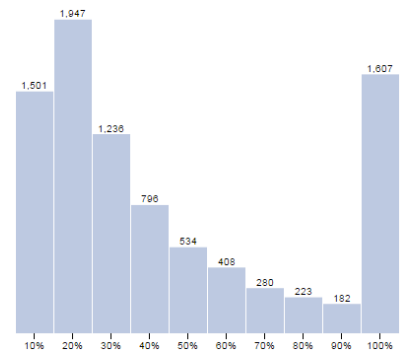

(d)
Figure 9: Visualization of particle transportation information using the VDS3 data set. (a) shows the VesselMap representation with the queried region, and two inlets marked by the blue and orange line segments. (b) shows the numbers of particles in the selected region over time. (c) and (d) show the histograms of lengths of time that the particles stay in the selected region released from the blue and orange inlets, respectively.

597 clicking a cell in the difference matrix, the row and column 598 containing that cell will be highlighted, as shown in (b). In 599 addition, the corresponding groups and particles will be high600 lighted in the VesselMap and the particle visualization as well, 601 as shown in (c) and (d), respectively.

\section{5. Results and Discussion}

Although our histogram interactions can be performed on 604 unsteady data, the current segmentation is based on the average 605 values computed at a given time step. Otherwise, segmenta606 tion may change over time and the relationships among groups 607 can be difficult to perceive. Thus, we only used steady fields 608 in this paper. Four data sets were used: vascular data set 1 609 (VDS1; one terminal aneurysm at middle cerebral artery), vas610 cular data set 2 (VDS2; two closely-spaced lateral aneurysms at 611 the internal carotid artery), vascular data set 3 (VDS3; one gi612 ant lateral aneurysm at the internal carotid artery), and vascular 613 data set 4 (VDS4; one lateral aneurysm at the internal carotid 614 artery). Their dimensions were $108 \times 60 \times 84,45 \times 63 \times 78$, $61572 \times 60 \times 72$, and $45 \times 92 \times 142$ respectively. The timing results 616 were collected on a PC with an Intel Core i7-3820 CPU running 617 at $3.6 \mathrm{GHz}, 16 \mathrm{~GB}$ main memory, and an nVidia Geforce 670 618 graphics card with 2 GB graphics memory. Even leveraging 619 the power of the GPU, the generation of the VesselMap dom620 inates the timing cost, as it requires multiple linear systems to 621 be solved. For the VDS1 containing 1095 blocks, the corre622 sponding VesselMap took 55.8 seconds to compute. The time 623 needed to calculate the VesselMaps of VDS2 (1482 blocks), 624 VDS3 (863 blocks), and VDS4 (1278 blocks) was 137.6, 35.2 


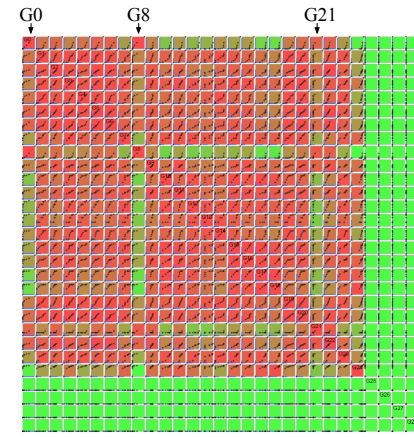

(a)

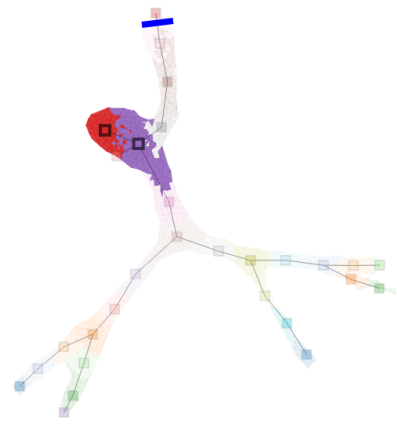

(c)

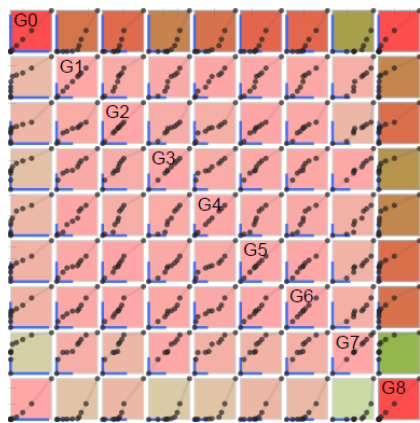

(b)

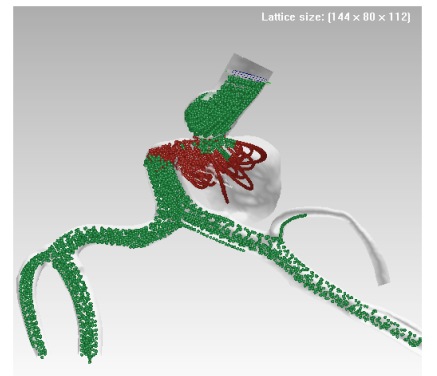

(d)
Figure 10: The relationships among velocity distributions of groups of blocks segmented according to their distances to the inlet. (a) shows the difference matrix. (b) is a zoom-in view focusing on the groups from $G 0$ to $G 8$. Cell $(G 0, G 8)$ is clicked, and row $G 0$ and column $G 8$ are highlighted. (c) and (d) are the segmentation result and particle visualization with groups $G 0$ and $G 8$ highlighted.

625 and 128.7 seconds, respectively. This performance is accept626 able since the VesselMap only needs to be computed once for 627 each data set. The histograms can be updated using the GPU in 628 real-time.

\section{5.1. Case Study: VDS1}

${ }_{630}$ We submitted a query to obtained particles with both large ${ }_{631}$ pressure and small velocity values. The results from the pro${ }_{632}$ posed system are shown in the first row of Figure 7. The Ves633 selMap and the particle visualization both show that the queried ${ }_{634}$ particles mostly resided in the aneurysm sac and those regions ${ }_{635}$ where vessel branches bifurcate. We observed from the inlet ${ }_{636}$ histogram that most of these particles were released from the ${ }_{637}$ positions corresponding to four bins. In the first row of Fig638 ure 8 , we also investigated the contribution of each position on 639 the seeding plane to the aneurysm sac. Note that some blocks 640 in the selected region are still in green because they do not con641 tain any particles (see Figure 7). Particle inlet visualization 642 indicates that most particles were released in a small region, 643 which is consistent with the inlet histogram. In the second row 644 of Figure 8, a segment of a vessel branch was selected, and 645 the contribution of each position in the inlet plane was some${ }_{646}$ what uniform. In the second row of Figure 7, a bin with small 647 age values and eight bins of inlet were selected. We observed 648 that the inlet positions corresponding to the particles with small 649 age values were mostly evenly distributed (see Figure 8). It 650 is also obvious that the velocities of these particles with small
651 age values are more uniformly distributed. Furthermore, among 652 those particles with small age values, there were more particles 653 with higher vorticity values, as compared with the histograms 654 in the first row of Figure 7. From the VesselMap, we observed 655 that most particles associated with small age were in one ves$656 \mathrm{sel}$ branch. This indicates that particles in the above-mentioned 657 vessel branch spent less time within the aneurysm sac.

658 In Figure 4 (b), the segmentation result of the VDS1 guided 659 by the vorticity showed that more groups were generated around 660 the neck of the aneurysm and around an inlet of a vessel branch. ${ }_{661}$ This indicates that the average vorticity values of blocks in 662 these regions varied more dramatically.

663 The segmentation guided by the distances to the inlet better ${ }_{664}$ preserved the shape of the vessel structure, as shown in Figure 4 665 (a). We used the difference matrix to analyze the differences 666 of velocity distributions among different groups. Note that the 667 groups that do not contain any particle will be considered as en668 tirely different with other groups, and the corresponding cells in 669 the difference matrix will all be colored in green. In Figure 10 670 (a), we observed that most green cells correspond to groups ${ }_{671} G 0, G 8$ and $G 21$, except the empty groups. This indicates that 672 the velocity in these three groups might be different from other 673 groups. Clicking at cell $(G 0, G 8)$ highlights row $G 0$ and column ${ }_{674} G 8$, as shown in Figure 10 (b). In cells $(G 0, G 0)$ and $(G 8, G 8)$, 675 points in the Q-Q plot were denser in the smaller value range. 676 Moreover, cells in row $G 0$ and $G 8$ showed that the Q-Q plot 677 lines mostly aligned horizontally when comparing these two 678 groups with other groups. These facts imply that particle veloc679 ities in these two groups were smaller than in the other groups. 680 By interacting with the difference matrix we found that $G 0$ and ${ }_{681} G 8$ belonged to the aneurysm region.

\section{5.2. Case Study: VDS2}

683 The vessel structure of the VDS2 can be perceived in Fig684 ure 11 (b) and (c). The first row of Figure 11 demonstrates the 685 query of particles with large age. The inlet histogram shows 686 that all particles with large age actually correspond to one bin, 687 which means that they were released at a very small region on 688 the seeding plane. From the VesselMap and the particle vi689 sualization, we observed that these particles went through the 690 aneurysm dome region, and their paths were close to the cen691 terline of the main vessel branch. In addition, as expected, these 692 particles mostly have small velocity values. In the second row ${ }_{693}$ of Figure 11, we select the aneurysm dome region for query. 694 We found that most particles in that region had moderate age 695 values. This confirms that the particles with large age were 696 mostly those that had already left the aneurysm dome. The in697 let histogram shows that the particles filling the aneurysm sac 698 actually came from multiple inlet positions. This is also con699 firmed by results presented in Figure 11 (c). However, compar700 ing the two inlet histograms in Figure 11 (a), we observed that 701 the particles that stayed within the aneurysm sac for a long time 702 were mostly released from a specific location. From the ve703 locity histograms, we found that the aneurysm dome even con704 tained more particles with slightly higher velocity values than 705 the particles with large age. This observation is consistent with 706 the fact that intra-aneurismal flow contains both high velocity 

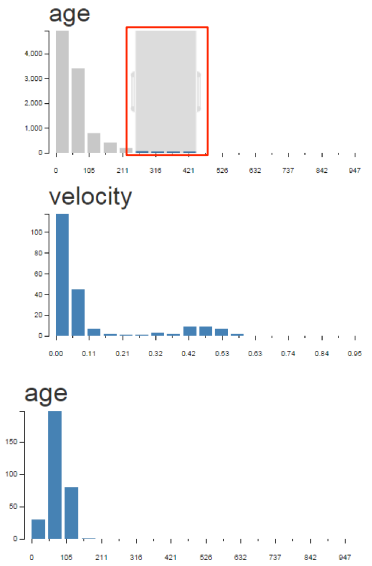

velocity

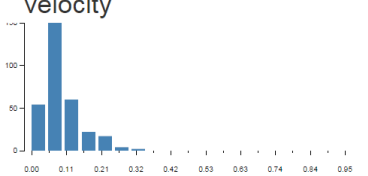

Figure 11: Visualization result using the VDS2 data ss aneurysm. (a) shows histogram visualization. (b) shows the VesselMap representation. (c) shows the visualization of particles with large age in the first row, and the inlet heat map associated with the aneurysm in the second row.

707 jet(s) and re-circulation zone(s) with low velocity. The pres708 sure and especially the WSS values mostly concentrate in a few 709 histogram bins, indicating more uniform distributions.

710 In the first row of Figure 12, we show the segmentation re711 sult guided by different properties. In (a), using the distances 712 to the inlet, we obtained the skeleton of the vessel structure. ${ }_{713}$ In (b), we can see four large groups segmented by age, which 714 are arranged similarly as the distances to the inlet. But there 715 are also some small groups, in which the particles are proba716 bly stuck and stay for a long time (i.e., long particle residence 717 time). In (c), we can see that three large groups align along the 718 main vessel branch. This indicates that there might be several 719 major paths with different velocities along the vessel. Note that 720 this finding is consistent with our discussion of the histograms 721 in Figure 11. In (d), we notice that the average vorticity value 722 varies in most of the vessel structure, but they are somewhat sta723 ble in the aneurysm dome and its immediate neighborhood. In 724 (e), we find that the WSS varies in the region near the aneurysm $725 \mathrm{sac}$. However, it is very stable inside the aneurysm, since the 726 aneurysm forms a single group. This can also be perceived in 727 the histogram shown in Figure 11.

728 We then investigate the relationship among groups, as shown 729 in the second row of Figure 12. For the groups segmented by 730 the distance to the inlet, we find that the age distribution of 731 groups $G 11$ and $G 12$ are different from other groups, as shown 732 in (f). The age for most groups cover a large range, with their 733 maximum values much larger than other values. But the age in ${ }_{734} G 11$ is distributed somewhat uniformly in a smaller range. Note 735 that $G 11$ corresponds to the top of the aneurysm region. This 736 indicates that the particles in this region follow similar paths 737 before, and have similiar age. (g) shows that the velocity in $G 2$ 738 and $G 12$ differs from other groups. We observe that $G 2$ con739 tains more particles with small velocity, which is common for
740 an aneurysm. (h) shows that the aneurysm region correspond741 ing to $G 2$ and $G 11$ contains more particles with small vortic742 ity. For the groups segmented by age, we find that the groups 743 form basically two clusters, considering the age distributions or 744 the velocity distributions, as shown in (i) and (j), respectively. 745 Groups $G 0$ to $G 4$ form one cluster, and the other groups form 746 the other one. Two groups from the same cluster have simi747 lar age and velocity distributions, but two groups from different 748 clusters are less similar. Note that the first cluster consists of 749 groups with more particles, containing the four major groups 750 in (b). It is likely that the isolated small groups have different 751 average age values from their neighbors due to velocity differ752 ences.

\section{5.3. Case Study: VDS3}

We compared the particle transport information using the 755 VDS3. The same quantity of particles was released for the 756 same duration of time at two different releasing locations, re757 spectively, as shown by the blue and orange lines in Figure 9 758 (a). The numbers of particles over time are displayed in (b), 759 where a curve corresponds to the inlet of the same color. Note 760 that, in (b), segments where the numbers of particles are zero 761 in the time-intensity curves were removed, so that those two 762 displayed curves were aligned by the time when their first re763 spective particles entered the selected region (see the red box 764 in (a)). We observed, from the curves in (b), that although the 765 orange plane was closer to the selected aneurysm sac region, 766 the peak density was actually lower, and the washout time was 767 shorter. Putting this into a practical perspective, we may tend 768 to choose a location that is closer to the aneurysm sac for agent 769 delivery. However, our result suggests that this intuition is ac770 tually incorrect.

In Figure 9 (c) and (d), the histograms corresponding to the 


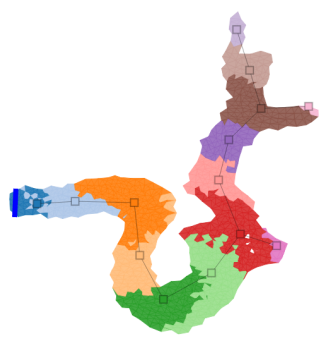

(a)

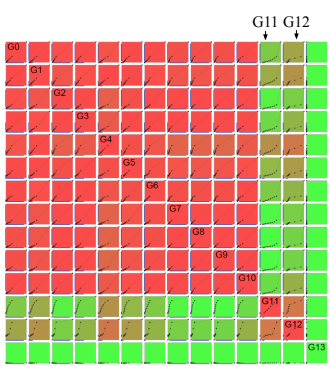

(f)

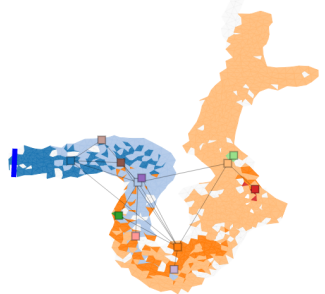

(b)

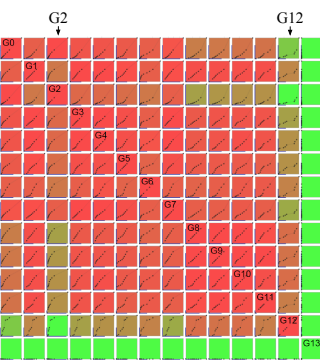

(g)

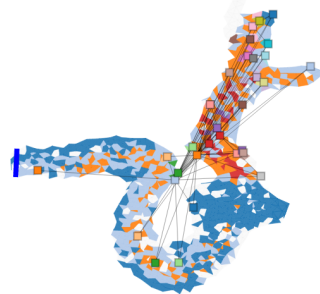

(c)

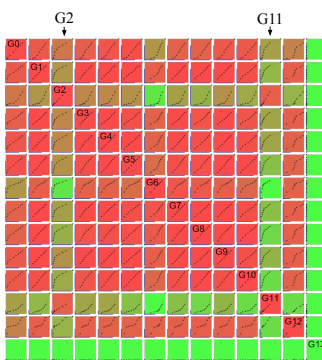

(h)

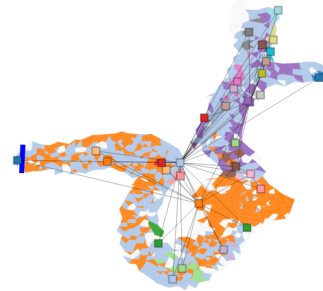

(d)

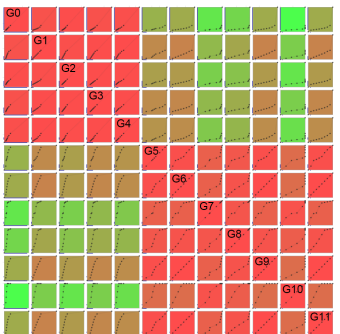

(i)

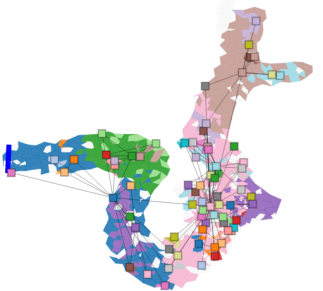

(e)

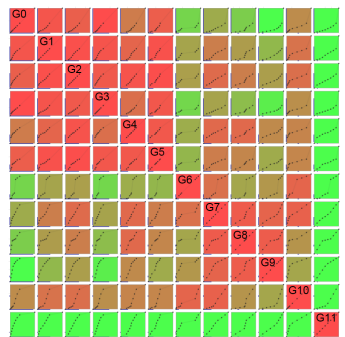

(j)

Figure 12: The segmentation result and group comparison using the VDS2 data set. First row: segmentation result guided by (a) distances to the inlet, (b) age, (c) velocity, (d) vorticity, and (e) WSS. Second row: group comparison of (f) age, (g) velocity, and (h) vorticity among groups segmented by distance to the inlet; and of (i) age and (j) velocity among groups segmented by age.

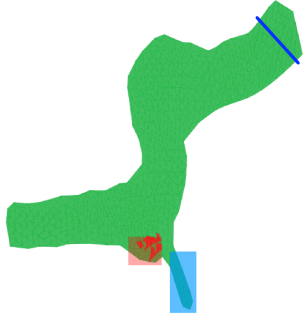

(a)

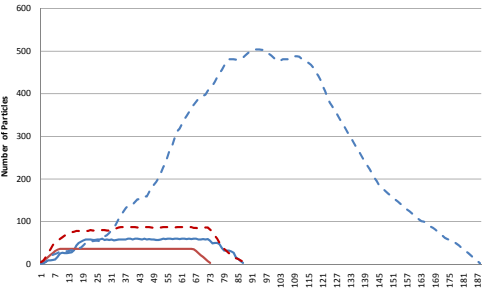

(b)
Figure 13: Visualization of particle transportation information using the VDS4 data set. (a) shows the VesselMap representation with two queried regions, indicated by the red and blue rectangles, respectively. (b) shows the numbers of particles in the two selected regions with two different vector fields over time. The red and blue lines correspond to the red and blue queried regions, respectively. And the solid and dashed lines correspond to Stents 1 and 2, respectively.

772 blue and orange releasing planes are shown. These two his773 tograms have similar shapes, indicating similar percentages of 774 particles staying for a certain amount of time. However, the ab775 solute numbers of particles in (d) were actually smaller, which 776 is consistent to the finding shown in (b). From the perspec777 tive of flow physics, a higher percentage of particles released 778 from the orange location/plane is directly diverted away from 779 the aneurysm, i.e., directly moving toward downstream without 780 entering the aneurysm. In short, the availability of such a tool 781 may enable biomedical engineers to improve their releasing lo782 cations for catheter-based drug delivery in a "patient-specific" 783 manner [36].

\section{5.4. Case Study: VDS4}

785 Unlike the VDS1, VDS2 and VDS3, each of which has a 786 single CFD simulation, VDS4 comes with two CFD simula787 tions using exactly the same vessel anatomy. Two CFD sim-
788 ulations represent two different flow-diverting stent configura789 tions: $20 \%$ (Stent 1 ) and $30 \%$ (Stent 2) metal coverage val790 ues. This case study helps us to evaluate the performance of 791 those two different stents in a "patient-specific" manner. In 792 general, a stent with a high metal coverage value (i.e., lower 793 porosity) diverts more flow entering the aneurysm sac (caus794 ing stable thrombosis) but also likely blocks blood flow pass795 ing through adjacent small arteries (e.g., ophthalmic artery in 796 this case). Now, by measuring the number of particles in the 797 aneurysm and the small vessel branch near the aneurysm, we 798 can evaluate the flow diverting effect while assessing how much 799 blood flow is spared for the ophthalmic artery. In Figure 13 800 (a), the queried aneurysm sac region and the small ophthalmic 801 artery are highlighted using red and blue semi-transparent rect802 angles, respectively. In Figure 13 (b), the number of particles 803 over time are displayed for the red and blue queried regions us804 ing the two different stents. All particles were released from 805 the same seeding plane for the same duration of time. It is easy 806 to see that a significant reduction of flow entering the aneurysm 807 sac (i.e., low particle numbers and longer wash-out time as in808 dicated by Figure 13) has been achieved by increasing the metal 809 coverage from $20 \%$ (Stent 1) to $30 \%$ (Stent 2). The increased 810 metal coverage in Stent 2 still allows the blood passage into the 811 ophthalmic artery.

\section{5.5. Discussion}

813 We would like to discuss three issues of the VesselMap rep814 resentation: preserving orientation, preserving branching order, 815 and the selection of target distances.

816 Preserving orientation. Preserving the orientation in the 817 2D VesselMap is almost impossible, as the 3D view can be ro818 tated. For example, in Figure 1 (a), the left and right branches 819 in the VesselMap correspond to the branches at the same side 820 of the $3 \mathrm{D}$ view, respectively. However, if the $3 \mathrm{D}$ structure is 


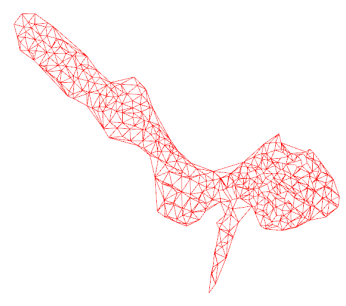

(a)

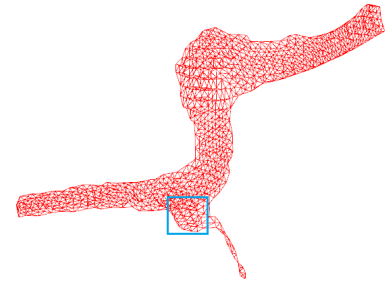

(b)
Figure 14: The VesselMap generated with different settings: (a) using only the geodesic distances for VDS3 data set and (b) using twice the geodesic distances for VDS4 data set.

821 viewed in an opposite direction, the orientation could be re822 versed. Actually, the orientation depends on the initial positions 823 of blocks. If a standard 3D view can be given, it is likely to gen824 erate the VesselMap with similar orientation, by using the 2D 825 projections of the blocks under the standard view as the initial 826 positions. But, if the $3 \mathrm{D}$ view is rotated, the orientation could 827 still be misleading. Therefore, unless the 2D VesselMap will be 828 adjusted according to the $3 \mathrm{D}$ view, it is not possible to preserve 829 the orientation.

${ }_{830}$ Preserving branching order. Our VesselMap representa${ }_{831}$ tion does not guarantee to preserve the order of vessel branches, 832 although the relative branching order may be preserved due to ${ }_{833}$ the distance preservation of different branches. For example, if 834 there exists one branch $b_{1}$ between two branches $b_{2}$ and $b_{3}$, the 835 distances between $b_{1}$ and $b_{2}$, and $b_{1}$ and $b_{3}$ should be small, 836 and the distance between $b_{2}$ and $b_{3}$ should be larger. To pre${ }_{837}$ serve the distance in the 2D VesselMap, the mapped branch $b_{1}$ 838 should still be in between of $b_{2}$ and $b_{3}$ and the branching or839 der is preserved. However, this may not always be the case. 840 For example, in Figure 1 (a), the two branches on the right are ${ }_{841}$ symmetric at the bifurcation point, and their order cannot be 842 captured by the distance between them. Also, similar to the ori843 entation, the branching order may also depend on the viewing 844 angle.

845 Selection of target distances. Our current approach uses 846 Euclidean distances for nearby blocks and geodesic distances 847 for the others. We argue that using both the two distances is 848 necessary to obtain the desired visualization. It is clear that 849 the different branches cannot be stretched out using only the 850 Euclidean distance. In addition, our experiment shows that us851 ing only the geodesic distance is also not enough, as shown 852 in Figure 14 (a). We can observe that the local shape is not 853 well preserved, and two bumpy regions appear on the vessel. In 854 contrast, in Figure 9, the VesselMap representation is smoother 855 along the boundary and preserves the curvy turns of the vessel. ${ }_{856}$ This is probably due to our approximation of geodesic distance 857 is not accurate when the Euclidean distance is small. There858 fore, for a small local neighborhood, it is still better to use only 859 the Euclidean distance; and for non-neighboring blocks, using 860 geodesic distance to stretch out the vessel branches seems to be 861 reasonable.

862 However, the current approach may be improved. In Fig863 ure 14 (b), we find that the aneurysm region highlighted in 864 the blue rectangle becomes larger and more standing-out when 865 the target distances for non-neighboring blocks are twice the
866 geodesic distances. This is likely due to the increase of the 867 force to stretch out the vessel branches (including aneurysms). 868 But the artifact is also obvious. It seems that the balance be869 tween the Euclidean distance and the geodesic distance is bro870 ken. The boundary of the vessel structure is not smooth. The 871 width of the main vessel branch varies in an unnatural way, and 872 serious distortion appears on the small branch, which seems to 873 be twisting. These indicate that the improvement demands fur874 ther research effort.

\section{6. Evaluation}

\section{6.1. Empirical Expert Evaluation}

877 Expert evaluations were done by one of the co-authors Dr. ${ }_{878} \mathrm{XXXX}$, who is a specialist in image-based biomechanical anal879 ysis. Dr. XXXX research interests include predicting and ana880 lyzing bio-flows for cardiovascular diseases. He has approx881 imately 10 years experience in cardiovascular flow analysis. 882 This evaluation aimed at providing an initial assessment on the 883 usefulness of the proposed system instead of quantitative re884 sults. The expert was asked to perform several tasks, includ885 ing querying regions with histograms, finding seeding locations 886 that fed the aneurysm, and selecting specific properties for seg887 mentation. These tasks were only designed to guide the expert 888 through the workflow of our approach. During the evaluation, 889 Dr. XXXX was informed to freely interact with our interface to 890 explore the VDS1 according to his own needs.

891 After learning the features of our interface, Dr. XXXX ex892 plored the VDS1 using our interface and provided the follow893 ing comments: "Overall, this is a good application and could 894 be particularly useful to collaborative research in transnational 895 biomedical device/technology design involving an engineer and 896 physician partnership. With this tool, engineers and physicians 897 can interactively explore and subsequently communicate exper898 imental and/or pre-clinical data over the internet. In addition, 899 this application does not significantly differ from typical web 900 applications. Thus, for ordinary users who are comfortable with 901 web browsing, it should be easy to learn. I definitely see its 902 potential use for flow visualization in a clinical setting given 903 further developments."

904 "In terms of effectiveness, the VesselMap allows users to 905 quickly select a region of interest in $2 \mathrm{D}$, because the flatten906 ing process will "evenly" map the $3 \mathrm{D}$ volume onto a $2 \mathrm{D}$ plane. 907 While in original 3D volumes, selecting a location inside the 908 volume is difficult because viewing of the desired location may 909 be blocked by objects in the background or foreground. A tech910 nical concern is that the flattening process of the VesselMap 911 may cause unrelated 3D points to look like spatial neighbors. 912 In terms of building connection between the 2D VesselMap and 913 the 3D vessel structure, users can brush across several branches 914 on the VesselMap, and observe the connection from highlighted 915 region in the corresponding 3D particle visualization. However, 916 since users may not be immediately aware of this, they should 917 be forced to perform this task in their learning stage. In ad918 dition, some visual landmarks can be placed on both the Ves919 selMap and the particle visualization, so that the connection can 920 be perceived more easily." 
${ }_{921}$ "Cross and Boolean filters are very useful. Currently, ev922 eryone looks at flow data based on his or her own experience. ${ }_{923}$ Thus, there is a good chance to overlook some important fea924 tures or correlations. In this application, once the queries are 925 set up, users can use certain combinations to quickly identify 926 areas of interest that meet all criteria. This helps to examine the 927 flow characteristics within and around a cerebral aneurysm in a 928 more comprehensive way."

${ }_{929}$ "The segmentation based on a criterion (e.g., distance, ve930 locity, etc.) will enable the user to quickly grasp spatial dis${ }_{931}$ tributions of the flow characteristics. Based on that, it should ${ }_{932}$ help the user select some important regions of interest for fur933 ther analyses. Some work may be needed to better organize ${ }_{934}$ or cluster the segmented regions. For instance, combined with 935 streamline visualization, segmented regions following a flow ${ }_{936}$ pathway will provide additional insight from the perspective of 937 bio-transportation. The difference matrix provides the overall ${ }_{938}$ correlation between different regions as well as a Q-Q plot. ${ }_{939}$ With the color-coded matrix, if we use age for segmentation 940 and identify groups with different velocity distributions, it is ${ }_{941}$ possible to guide the user to flow stagnation regions. Thus, this 942 segmentation scheme could potentially help users identify re943 gions which contain interesting flow features."

\section{6.2. User Study}

945 We recruited six unpaid graduate students for a quantita946 tive study: four majoring in biomedical engineering, and the 947 other two majoring in computer science. Three of those four 948 biomedical engineering students have (1-2 years) experience of 949 using commercial or open-source software (e.g., Ensight and 950 Paraview) for bio-flow visualization. Therefore, these three 951 students were considered to be the advanced users (AU). The 952 remaining three students are either knowledgeable in vascular 953 structures or visualization, but they never used visualization 954 tools for vascular flow before this study. They were consid955 ered as beginning users (BU). The user study was conducted 956 in a lab using the same PC with only one participant presented 957 at one time. The PC has a 27 -inch monitor with $1920 \times 800$ 958 resolution, where the user study interface occupies an area of $9591800 \times 600$ pixels. Each study took about an hour to complete. 960 The study consists of three stages: an exploration stage, a test 961 stage, and a rating stage. The exploration stage allowed users to 962 get familiar with our interface and the vascular structure in the ${ }_{963}$ VDS1, which was used in the test stage as well. In the test stage, 964 users were asked to reproduce previous selection results using 965 both the VesselMap and the traditional punching selection. The 966 punching operation is commonly used in clinical workstations 967 by clinicians and biomedical engineers. It selects a polygonal 968 region in the screen space so that all voxels projected to that re969 gion are selected. Users may rotate the scene and select again, 970 so that only the intersection of the two selections remains, and 971 the undesired voxels are excluded. This operation will be re972 peated several times so that users can gradually refine the se973 lection. Finally, in the rating stage, users were asked to provide 974 ratings to several statements.

975 The major goal of this user study is to compare our Ves976 selMap and the punching selection in terms of the effectiveness
Table 1: Averages and standard deviations of task completion time and accuracies for different user groups. "AU" and "BU" were advanced users and beginning users, respectively. Timing is measured in seconds, and accuracy is presented in percentage.

\begin{tabular}{|l|l|r|r|r|r|}
\hline \multicolumn{2}{|c|}{} & \multicolumn{2}{|c|}{ timing } & \multicolumn{2}{c|}{ accuracy (\%) } \\
\cline { 3 - 6 } \multicolumn{2}{|c|}{} & VesselMap & punch & VesselMap & punch \\
\hline \multirow{2}{*}{ AU } & average & 26.0 & 64.8 & 89.2 & 81.4 \\
& std. dev. & 20.2 & 73.5 & 7.8 & 8.8 \\
\hline \multirow{2}{*}{ BU } & average & 31.3 & 72.8 & 91.4 & 77.1 \\
& std. dev. & 19.6 & 36.6 & 8.0 & 11.2 \\
\hline \multirow{2}{*}{ All } & average & 28.7 & 68.8 & 90.3 & 79.2 \\
& std. dev. & 20.0 & 57.9 & 7.9 & 10.3 \\
\hline
\end{tabular}

977 to select a specific region. Two factors were considered: effi978 ciency and reproducibility, measured by the time used and ac979 curacy to reselect a set of blocks given one or two screenshots 980 of a previous selection result. Users were informed that the 981 accuracy was more important than the completion time. A pro982 gram was designed specifically for this user study. For selection 983 using the VesselMap, one screenshot of the previous selection 984 would be given; and for the punching selection, two screenshots 985 would be given: one in a manually selected direction that best 986 demonstrated the region to be selected, and the other in the an987 terior direction as a standard view. For fair comparisons, the 988 selection for both methods were performed at the block level.

989 We selected five regions and generated the screenshots for 990 both the VesselMap and the punching selection. For each task, 991 users were presented the screenshots of one selected region. ${ }_{992}$ Users would need to reselect that region to finish the task. Each 993 user was required to perform three rounds of selection tasks. ${ }_{994}$ In each round, they would need to perform five different tasks 995 using the VesselMap followed by five using the punching selec996 tion. Therefore, each user performed $2 \times 5 \times 3$ selection tasks 997 in total. Note that the tasks were the same for each round, but 998 they appeared in different orders.

999 Timing and Accuracy. Since the completion time and ac000 curacy might be related, we applied MANOVA (multivariate001 ANOVA) to investigate the overall difference, and ANOVA to 1002 study the difference of each individual variable. For MANOVA, ${ }_{003}$ Wilk's Lambda test was used and both the completion time and 004 accuracy were considered at the same time. We used the sig005 nificance level of 0.05 for all tests, and our findings were as 1006 follows.

MANOVA found significant differences of timing and accu1008 racy for the VesselMap and the punching selection with the $p$ 1009 value of $2 \times 10^{-16}$. The differences of timing and accuracy us010 ing these two methods were significant using ANOVA as well, 1011 with the $p$-values of $2 \times 10^{-9}$ and $1 \times 10^{-13}$, respectively. In 012 Table 1, we observe that using the VesselMap, the completion 1013 time is much shorter and accuracies are higher for both the AU 1014 and BU groups. This indicates that our VesselMap improves 015 the efficiency and reproducibility over the punching selection. 1016 ANOVA also suggested a significant difference of comple- 


\begin{tabular}{|l|l|l|l|l|}
\hline & $\mathrm{Q} 1$ & $\mathrm{Q} 2$ & $\mathrm{Q} 3$ & $\mathrm{Q} 4$ \\
\hline average & 4.33 & 2.67 & 4.67 & 4.17 \\
\hline std. dev. & 0.51 & 1.03 & 0.51 & 0.98 \\
\hline
\end{tabular}

1066 The rating is a five-point scale, where "5" means strongly 1067 agree and " 1 " means strongly disagree. The rating results are 1068 shown in Table 2. The averages of ratings for Q1, Q3 and Q4 1069 were all higher than 4 , with the highest being 4.67 for Q3. The 1070 high rating for Q1 and Q4 indicated our automatic viewpoint se1071 lection using the VesselMap produced satisfactory results. The 1017 tion time for the three rounds with the $p$-value of 0.040. The 1072 rating for Q3 indicated that users agreed that repeating a view1018 overall averages of completion time for the three rounds were 1073 point using the VesselMap was easy. The rating for Q2 (2.67) 1019 59.3, 43.5 and 43.4 seconds, respectively. The average com- 1074 was low, meaning users did not agree that repeatedly select1020 pletion time for both the VesselMap (32.6, 27.4 and $26.1 \mathrm{sec}-1075$ ing the same viewpoint is easy by manipulating the 3D view. 1021 onds) and the punching selection (86.1, 59.7 and 60.6 seconds) 1076 We then applied the non-parametric Kruskal-Wallis (KW) test 1022 show the same trend. We found that the differences among the 1077 to study the rating difference between Q2 and Q3. Note that, 1023 completion time during the three rounds were smaller using the 1078 unlike in the analysis of the timing and accuracy, we did not 1024 VesselMap, which might indicate that it is easier to repeat a task 1079 use t-test for the rating, since the sample size was small and the 1025 with the VesselMap. However, no significant difference of ac- 1080 rating did not follow a normal distribution. The KW-test sug1026 curacy was found for the three rounds. The overall accuracies 1081 gested a significant difference with the $p$-value of 0.005 . This 1027 for the three rounds were actually very closed, being 85.1\%, 1082 indicated that repeating a viewpoint using the VesselMap was $102884.7 \%$ and $84.5 \%$, respectively. Users were able to perform the 1083 easier.

1029 tasks with high accuracy, but it just required more time for the 1030 first time.

${ }_{1031}$ We further investigated the impact of expertise using these

1084 7. Conclusions and Future Work

1032 two methods. The averages and standard errors are shown in 1085 We present VesselMap, a web interface to explore multivari${ }_{1033}$ Table 1. We did not find any significant difference of timing 1086 ate vascular data sets. The web interface provides great com1034 using the VesselMap between AU (averaged at 26.0 seconds) 1087 patibility for users working on different platforms. Interaction 1035 and BU (averaged at 31.3 seconds) groups with the $p$-value of 1088 with histograms allows users to quickly identify regions meet1036 0.210. For accuracy, no significant difference was found as well 1089 ing certain criteria. The 2D VesselMap representation provides 1037 (with the $p$-value of 0.193), and the accuracies for AU (89.2\%) 1090 not only an overview but also an efficient method to select re1038 and BU (91.4\%) groups were very close. For the punching se- 1091 gions of interest. In addition, local behaviors of different prop1039 lection, although the difference of average completion time was 1092 erties can be discovered effectively using the segmentation and 1040 larger (64.9 seconds for AU and 72.8 seconds for BU), ANOVA 1093 group comparison features.

1041 did not suggest any significant difference (the $p$-value is 0.508). $1094 \quad$ There are challenges to be solved in the future as well. First, 1042 This was likely due to the larger variation of the completion 1095 although the discontinuity produced by the flattening process 1043 time, as shown in Table 1. In terms of accuracy, ANOVA found 1096 from 3D volumes to 2D maps is unavoidable, we might post1044 a significant difference between $\mathrm{AU}(81.3 \%)$ and BU (77.1\%) 1097 process the user selection and enforce the selected blocks to 1045 groups with the $p$-value of 0.050 . This indicated that the punch- 1098 be spatial neighbors in the 3D volume. The same procedure 1046 ing selection required more experience from users to perform 1099 can be applied when highlighting a region, so that the blocks 1047 accurately.

1100 look continuous on the VesselMap. Second, the performance of 1048 Overall, the results show that our VesselMap is better in 1101 generating the VesselMap can be improved. The constraints in 1049 terms of efficiency and reproducibility. Furthermore, it is likely 1102 the linear system are enforced on every two blocks. We may 1050 that the VesselMap has lower requirements on user expertise 1103 use the centers of groups segmented by distances to the inlet as 1051 and learning effort.

1104 anchor points, and only enforce the constraints on every two an1052 Ratings. We observed during the user study that the diffi- 1105 chor points and neighboring blocks. In this way, the size of the ${ }_{1053}$ culty of using the punching selection resided in selecting the 1106 linear system to be solved can be greatly reduced. Third, the 1054 viewing direction shown in the screenshots, which might be 1107 automatic viewpoint selection can be significantly improved. 1055 solved by our automatic viewpoint selection. For the rating 1108 The current approach uses a weighted sum of the three princi1056 questions, we asked users to freely interact with the interface 1109 pal components as the viewing direction, which only considers 1057 to select viewpoints using the VesselMap and rate the follow- 1110 the spatial distribution of selected blocks. A more sophisticated 1058 ing statements:

1111 solution may evaluate the information revealed under a certain 1112 viewpoint, considering both occlusions among blocks and flow

- Q1. The automatically selected viewpoint is close to the ideal viewpoint. - Q2. It is easy to repeat a viewpoint by translation and rotation in 3D.

- Q3. It is easy to repeat a viewpoint using the VesselMap. 1117 tended to handle unsteady data sets. For example, the average

- Q4. It is easy to adjust the automatically selected view- 
1118 value in a block over a time span can be used to guide the seg- 1185 [18] Marino J, Kaufman A. Planar visualization of treelike structures. IEEE 1119 mentation. Finally, many practical features can be further de- 1186 1120 veloped based on the VesselMap. For example, after placing a ${ }^{1187}$ 1121 seeding plane using the VesselMap, users may further rotate the ${ }_{1189}^{1188}$ 1122 plane for a desired orientation. Users may place labels on the 1190 ${ }_{1123}$ VesselMap, and our system can generate a report highlighting ${ }^{1191}$ 1124 the labeled regions in the 3D volume, together with comments. ${ }_{1193}^{1192}$ 1125 We may also allow users to measure the volumetric size of the ${ }_{1194}^{1193}$ 1126 selected region, etc. Transactions on Visualization and Computer Graphics 2016;22(1):90615 .

[19] Neugebauer M, Gasteiger R, Beuing O, Diehl V, Skalej M, Preim B. Map displays for the analysis of scalar data on cerebral aneurysm surfaces. Computer Graphics Forum 2009;28(3):895-902.

[20] Won JH, Jeon Y, Rosenberg J, Yoon S, Rubin G, Napel S. Uncluttered single-image visualization of vascular structures using gpu and integer programming. IEEE Transactions on Visualization and Computer Graphics 2013;19(1):81-9.

1195 [21] Neugebauer M, Lawonn K, Beuing O, Preim B. Automatic genera1196 tion of anatomic characteristics from cerebral aneurysm surface mod1197 els. International Journal of Computer Assisted Radiology and Surgery $1198 \quad 2013 ; 8(2): 279-89$.

\section{References} 40
[1] Brass LM, Lichtman JH, Wang Y, Gurwitz JH, Radford MJ, Krumholz ${ }_{1200}^{1199}$ HM. Intracranial hemorrhage associated with thrombolytic therapy for ${ }_{120}$ elderly patients with acute myocardial infarction results from the cooper- 1202 ative cardiovascular project. Stroke 2000;31(8):1802-11.

[2] Molyneux AJ, Cekirge S, Saatci I, Gál G. Cerebral aneurysm multicenter european onyx (CAMEO) trial: results of a prospective observational study in 20 european centers. American Journal of Neuroradiology 2004;25(1):39-51.

3] Guan YS, Sun L, Zhou XP, Li X, Fei ZJ, Zheng XH, et al. Polyvinyl alcohol and gelatin sponge particle embolization of splenic artery pseudoaneurysm complicating chronic alcoholic pancreatitis. World Journal of Gastroenterology 2005;11(17):2684-6.

4] van Rooij WJ, Sluzewski M, Beute GN. Brain AVM embolization with onyx. American Journal of Neuroradiology 2007;28(1):172-7.

[5] Hahn HK, Preim B, Selle D, Peitgen HO. Visualization and interaction techniques for the exploration of vascular structures. In: Proceedings of IEEE Visualization Conference. 2001, p. 395-402.

[6] Höhne KH, Pflesser B, Pommert A, Riemer M, Schubert R, Schiemann T, et al. A realistic model of the inner organs from the visible human data. In Proceedings of International Conference on Medical Image Computing and Computer-Assisted Intervention. 2000, p. 776-85.

[7] Felkel P, Wegenkittl R, Bühler K. Surface models of tube trees. In: Proceedings of Computer Graphics International. 2004, p. 70-7.

[8] Oeltze S, Preim B. Visualization of vasculature with convolution surfaces: Method, validation and evaluation. IEEE Transactions on Medical Imaging 2005;24(4):540-8.

[9] Lorensen WE, Cline HE. Marching cubes: A high resolution 3D surface construction algorithm. In: Proceedings of ACM SIGGRAPH Conference. 1987, p. 163-9.

0] Läthé G, Lindholm S, Lenz R, Persson A, Borga M. Automatic tuning of spatially varying transfer functions for blood vessel visualization. IEEE Transactions on Visualization and Computer Graphics 2012;18(12):2345-54

11] Mistelbauer G, Varchola A, Bouzari H, Starinsky J, Köchl A, Schernthaner R, et al. Centerline reformations of complex vascular structures. In: Proceedings of IEEE Pacific Visualization Symposium. 2012, p. 233

2] Schumann C, Oeltze S, Bade R, Preim B, Peitgen HO. Model-free surface visualization of vascular trees. In: Proceedings of Joint Eurographics IEEE VGTC Symposium on Visualization. 2007, p. 283-90.

13] Kanitsar A, Fleischmann D, Wegenkittl R, Gröller ME. Diagnostic relevant visualization of vascular structures. In: Bonneau GP, Ertl T, Nielson G, editors. Scientific Visualization: The Visual Extraction of Knowledge from Data. 2006, p. 207-28.

4] Kretschmer J, Preim B, Stamminger M. Bilateral depth filtering for enhanced vessel reformation. In: Proceedings of Eurographics Workshop on Visual Computing for Biology and Medicine. 2014, p. 117-26.

5] Mistelbauer G, Morar A, Varchola A, Schernthaner R, Baclija I, Köchl A, ${ }^{1246}$ et al. Vessel visualization using curvicircular feature aggregation. Computer Graphics Forum 2013;32(3):231-40.

[16] Borkin M, Gajos K, Peters A, Mitsouras D, Melchionna S, Rybicki $\mathrm{F}$, et al. Evaluation of artery visualizations for heart disease diagnosis. IEEE Transactions on Visualization and Computer Graphics 2011;17(12):2479-88.

7] Zhu L, Haker S, Tannenbaum A. Flattening maps for the visualization of multibranched vessels. IEEE Transactions on Medical Imaging 2005;24(2):191-8.
22] van Pelt R, Bescós JO, Breeuwer M, Clough RE, Gröller ME, ter Haar Romeny B, et al. Exploration of 4D MRI blood flow using stylistic visualization. IEEE Transactions on Visualization and Computer Graphics 2010;16(6):1339-47.

[23] Köhler B, Gasteiger R, Preim U, Theisel H, Gutberlet M, Preim B. Semiautomatic vortex extraction in 4D PC-MRI cardiac blood flow data using line predicates. IEEE Transactions on Visualization and Computer Graphics 2013;19(12):2773-82

7 [24] van Pelt R, Bescós JO, Breeuwer M, Clough RE, Gröller ME, ter Haar Romeny B, et al. Interactive virtual probing of 4D MRI bloodflow. IEEE Transactions on Visualization and Computer Graphics 2011;17(12):2153-62.

1 [25] Born S, Markl M, Gutberlet M, Scheuermann G. Illustrative visualization of cardiac and aortic blood flow from 4D MRI data. In: Proceedings of IEEE Pacific Visualization Symposium. 2013, p. 129-36.

[26] Oeltze S, Lehmann DJ, Kuhn A, Janiga G, Theisel H, Preim B. Blood flow clustering and applications in virtual stenting of intracranial aneurysms. IEEE Transactions on Visualization and Computer Graphics 2014;20(5):686-701.

[27] Angelelli P, Hauser H. Straightening tubular flow for side-by-side visualization. IEEE Transactions on Visualization and Computer Graphics 2011;17(12):2063-70.

[28] Straka M, Köchl A, Červeňanský M, Šrámek M, Fleischmann D, La Cruz A, et al. The VesselGlyph: Focus \& context visualization in CTangiography. In: Proceedings of IEEE Visualization Conference. 2004, p. $385-92$.

[29] Gasteiger R, Neugebauer M, Beuing O, Preim B. The FLOWLENS: A focus-and-context visualization approach for exploration of blood flow in cerebral aneurysms. IEEE Transactions on Visualization and Computer Graphics 2011;17(12):2183-92.

[30] Lawonn K, Gasteiger R, Preim B. Adaptive surface visualization of vessels with animated blood flow. Computer Graphics Forum 2014;33(8):1627.

2 [31] Lawonn K, Glaßer S, Vilanova A, Preim B, Isenberg T. Occlusion-free blood flow animation with wall thickness visualization. IEEE Transactions on Visualization and Computer Graphics 2015;22(1):728-37.

5 [32] Kanitsar A, Fleischmann D, Wegenkittl R, Felkel P, Gröller ME. CPR curved planar reformation. In: Proceedings of IEEE Visualization Conference. 2002, p. 37-44.

238 [33] Singh G, Memoli F, Carlsson G. Topological methods for the analysis of 1239 high dimensional data sets and 3D object recognition. In: Proceedings of Eurographics Symposium on Point-Based Graphics. 2007, p. 91-100.

[34] Hatcher A. Algebraic Topology. Cambridge University Press; 2002

[35] Chambers JM, Cleveland WS, Tukey PA, Kleiner B. Graphical Methods for Data Analysis. Duxbury Press; 1983.

[36] Kavanagh CA, Rochev YA, Gallagher WM, Dawson KA, Keenan AK. Local drug delivery in restenosis injury: thermoresponsive co-polymers as potential drug delivery systems. Pharmacology \& therapeutics 2004;102(1):1-15. 


\section{VesselMap: A Web Interface to Explore Multivariate Vascular Data}
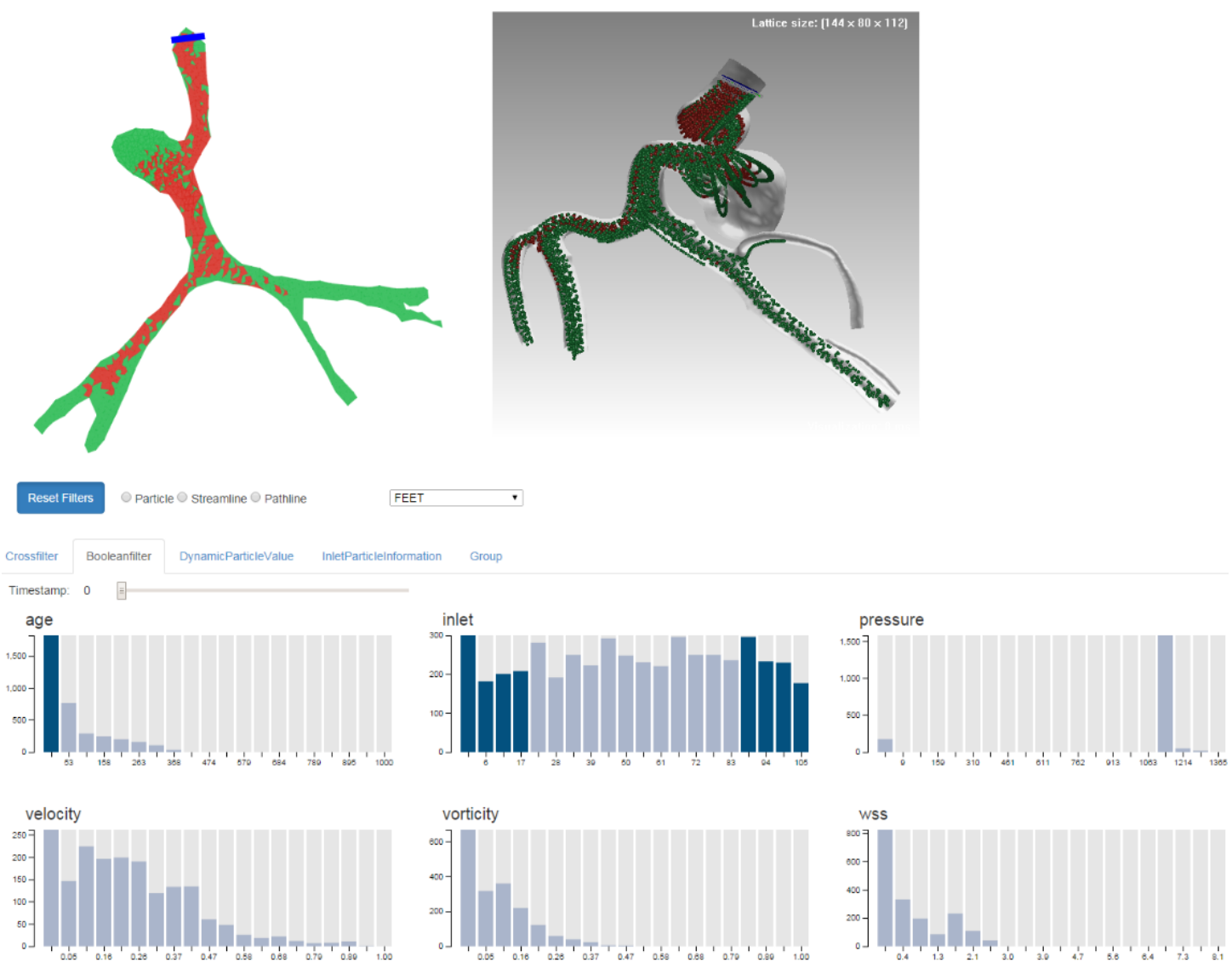

VesselMap provides a 2D representation of vessel structures for efficient interaction together with a series of queries for dynamic knowledge discovery of vascular data sets during particle tracing. 